\title{
Effects of Continuum Breakdown on the Surface Properties of a Hypersonic Sphere
}

\author{
Timothy D. Holman* and Iain D. Boyd \\ University of Michigan, Ann Arbor, Michigan 48109
}

DOI: $10.2514 / 1.43509$

\begin{abstract}
This study investigates the effects of continuum breakdown on the surface aerothermodynamic properties (pressure, shear, heat transfer rate) of a sphere in Mach 10, 25, and 45 flows of nitrogen gas in regimes varying from continuum to rarefied gas. A rotational energy relaxation model is employed in the computational fluid dynamics code and is tested to confirm its accuracy. As the global Knudsen number is increased, from continuum flow to a rarefied gas, the amount of continuum breakdown seen in the flow and on the surface is increased. This increase in continuum breakdown affects the surface properties, such that an increase in the differences between computational fluid dynamics and direct simulation Monte Carlo method is observed. As the Mach number is increased, the amount of continuum breakdown observed in the flow is increased, but the gradient length local Knudsen number stays approximately constant. Even though the amount of continuum breakdown has increased, the difference between computational fluid dynamics and direct simulation Monte Carlo method remains relatively constant. The last part of this study compares the results of the sphere with that of the analogous cylinder case. At the same global Knudsen number, the differences in the surface properties between computational fluid dynamics and direct simulation Monte Carlo method increase when the simulation is run axisymmetrically.
\end{abstract}

\section{Nomenclature}

$C_{q}$

$C_{\tau}$

$\bar{c}$

E

$K n_{\mathrm{GLL}}$

$K n_{\infty}$

$k$

$L$

M

$m$

$m^{*}$

$P$

$p$

$q$

Re

$T$

$U$

Z

$\gamma$

$\zeta$

$\lambda$

$\mu$

$v$

$\rho$

$\sigma$

$\tau$

$\omega$

$=$ pressure coefficient

$=$ heat flux coefficient

$=$ shear coefficient

$=$ mean speed, $\mathrm{m} / \mathrm{s}$

$=$ molecular diameter, $\mathrm{m}$

$=$ energy per unit volume, $\mathrm{J} / \mathrm{m}^{3}$

$=$ global Knudsen number

$=$ characteristic length, $\mathrm{m}$

$=$ Mach number

$=$ mass, $\mathrm{kg}$

$=$ relative mass, $\mathrm{kg}$

$=$ average probability

$=$ pressure, $\mathrm{Pa}$

$=$ heat transfer rate, $\mathrm{W} / \mathrm{m}^{2}$

$=$ Reynolds number

$=$ translational temperature, $\mathrm{K}$

$=$ freestream velocity, $\mathrm{m} / \mathrm{s}$

$=$ ratio of specific heats

$=$ internal degrees of freedom

$=$ mean free path, $\mathrm{m}$

$=$ viscosity, $\mathrm{Pa} \cdot \mathrm{s}$

$=$ mean collision rate, $1 / \mathrm{s}$

$=$ density, $\mathrm{kg} / \mathrm{m}^{3}$ accommodation coefficient
$=$ gradient length local Knudsen number

$=$ Boltzmann constant, $1.38 \times 10^{-23} \mathrm{~J} / \mathrm{K}$

$=$ collision number

$=$ collision cross section, $5.81 \times 10^{-21} \mathrm{~m}^{2}$,

$=$ shear stress, $\mathrm{Pa}$, relaxation time, $\mathrm{s}$

$=$ variable hard sphere temperature exponent

Presented as Paper 3928 at the 40th AIAA Thermophysics Conference, Seattle, WA, 23-26 June 2008; received 29 Jan. 2009; revision received 2 June 2009; accepted for publication 14 June 2009. Copyright $\odot 2009$ by the American Institute of Aeronautics and Astronautics, Inc. All rights reserved. Copies of this paper may be made for personal or internal use, on condition that the copier pay the $\$ 10.00$ per-copy fee to the Copyright Clearance Center, Inc., 222 Rosewood Drive, Danvers, MA 01923; include the code 0887-8722/ 09 and $\$ 10.00$ in correspondence with the CCC.

${ }^{*}$ Graduate Student, Department of Aerospace Engineering. Member AIAA.

${ }^{\dagger}$ Professor, Department of Aerospace Engineering. Associate Fellow AIAA.

$\begin{array}{ll}\text { Subscripts } & \\ \text { cont } & =\text { continuum } \\ \mathrm{LT} & =\text { Landau-Teller } \\ p & =\text { park } \\ \text { part } & =\text { particle } \\ r & =\text { rotation } \\ \text { ref } & =\text { reference value } \\ v & =\text { vibration } \\ \infty & =\text { freestream }\end{array}$

\section{Introduction}

W ITH a renewed desire to send humans back to the moon and beyond there is a need for accurate studies of the flow behavior over hypersonic vehicles to precisely determine how they will perform when entering an atmosphere. Experiments and flight tests are extremely difficult and expensive, and so there is need for computational models that can be used for design and development of hypersonic vehicles.

A hypersonic vehicle that is entering an atmosphere will go through many different flow regimes due to the change in atmospheric density with altitude. The flow can be characterized by the following Reynolds and the Knudsen numbers:

$$
R e=(\rho U L) / \mu \quad K n=(\lambda / L) \propto(1 / \rho L)
$$

The flow can be considered continuum when the Knudsen number is much less than one. In the continuum regime, flows should be simulated using traditional computational fluid dynamics techniques by numerically solving the Navier-Stokes equations. However, when the Knudsen number becomes larger, the continuum assumption in the Navier-Stokes equations starts to breakdown. This is due to the fact that these equations are derived from kinetic theory based on the assumption of small perturbations from an equilibrium velocity distribution function [1]; therefore, computational fluid dynamics (CFD) only works in near equilibrium flows. When the Knudsen number grows, only a noncontinuum technique can be used, such as the direct simulation Monte Carlo (DSMC) method [2]. DSMC is a Monte Carlo particle method for simulating nonequilibrium gas flows. DSMC is required for accurate flow analysis of hypersonic rarefied flows for which the continuum flow equations are invalid and can be used in any dilute gas flow. Unfortunately, DSMC is about an order of magnitude more expensive than traditional CFD methods 
and becomes prohibitively expensive at low Knudsen numbers. Note that even if the global flow behaves as a continuum there may still be parts of the flow that locally act as a rarefied flow, if the local length scale is very small or the local density is low. For example a hypersonic blunt body can create a locally rarefied flow in the shock, the boundary layer, and the wake of the body. As a result, neither CFD nor DSMC can provide a complete computational model across all regimes of a hypersonic vehicle.

One solution to this problem is a hybrid code that uses CFD and DSMC methods to accurately and efficiently simulate a hypersonic flow. This hybrid code can solve the Navier-Stokes equations when the flow is considered to be a continuum, but it can switch to a DSMC method when the flow is considered rarefied such as in a shock wave. This process involves some way of finding when the physics of the simulation provided by the CFD method deviates from physical results; this is performed with the continuum breakdown parameter. This continuum breakdown parameter will notify the code that the CFD method can no longer be employed and that DSMC needs to be used to correctly simulate the flowfield. Bird [3] has proposed a continuum breakdown parameter, $P$ :

$$
P=\frac{1}{v}\left|\frac{\mathrm{D}(\ln \rho)}{\mathrm{D} t}\right|=M \sqrt{\frac{\pi \gamma}{8}} \frac{\gamma}{\rho}\left|\frac{\mathrm{d} p}{\mathrm{~d} s}\right|
$$

in which $M$ is the local Mach number and $s$ is the distance along a streamline. Bird found that a value of $P$ over 0.05 is an indication of continuum breakdown. This parameter predicts breakdown well in expansion flows but has problems when the Mach number is small. Because $P$ is directly proportional to Mach number, it will tend to zero as the Mach number goes to zero despite the amount of breakdown in the flow. Boyd et al. [4] suggested the use of the maximum gradient length local Knudsen number as a continuum breakdown parameter:

$$
K n_{\mathrm{GLL}}=\frac{\lambda}{Q}\left|\frac{\mathrm{d} Q}{\mathrm{~d} l}\right|
$$

in which the derivative is taken in the direction of maximum gradient and $Q$ is a variable of interest such as density, temperature, or pressure. It has been found that a value of $K n_{\mathrm{GLL}}$ above 0.05 indicates continuum breakdown has occurred. Camberos et al. [5] recommended the use of entropy production rate as a continuumbreakdown parameter. There have been several other breakdown parameters proposed: Tiwari suggested a breakdown parameter [6] as well as the $B$ parameter suggested by Garcia et al. [7].

To be able to design a hypersonic vehicle it is important to understand how continuum breakdown affects the surface conditions such as heat flux, pressure, and shear stress. These surface conditions determine the aerodynamic and thermodynamic performance of a reentry vehicle. A previous study by Lofthouse et al. looked at the effect of breakdown on the surface properties of a 12-in.-diam 2-D cylinder in a Mach 10 flow of argon [8]. A more recent study by Lofthouse et al. examined the effects of velocity slip and temperature jump at the surface of a 2-D 12-in.-diam cylinder in Mach 10 and 25 flow of argon [9]. Another study by Lofthouse et al. investigated Mach 10 and 25 nitrogen flow over a cylinder [10]. All of these studies were conducted over a range of Knudsen numbers, 0.0020.25 , in which the characteristic length is taken to be the diameter of the cylinder. The present study will continue to examine the continuum breakdown phenomenon in Mach 10, 25, and 45 flows of nitrogen over a 12-in.-diam sphere with the same global Knudsen number range as the previous investigations. This gives a range of flow conditions from continuum to rarefied gas. The purpose of this work is to accurately characterize the effects of continuum breakdown. This has to be preformed by starting out with simple simulations and then adding complexity to determine individual effects on continuum breakdown. The work done by Lofthouse et al. started this effort by characterizing breakdown over a 2-D cylinder in flows of argon and nitrogen. This study continues to characterize breakdown by considering a geometry that is more representative of actual reentry vehicles such as capsules. Although this study is concerned with nitrogen it does not include chemical reactions, that will be included in future studies.

This paper will discuss the simulation procedures as well as the computational models used to run the simulations. Selected results are presented in four parts. First, the results of the rotational energy model introduced into CFD are given to determine the accuracy of the model. Second, the results of the simulations from the whole range of global Knudsen numbers at a Mach number of 10 are discussed. Third, the results from the simulations of a global Knudsen number of 0.01 at all three Mach numbers are presented. Fourth, a comparison is made of the results from the sphere and cylinder simulations. Finally, conclusions and future work are discussed.

\section{Background}

This study examines the continuum breakdown phenomenon in flows of nitrogen over a 12-in.-diam sphere at Mach numbers of 10, 25 , and 45 . The freestream temperature is $200 \mathrm{~K}$ giving freestream velocities of $2883.5,7208.75$, and $12975.75 \mathrm{~m} / \mathrm{s}$ for the three different Mach numbers. The surface of the sphere has fixed temperatures of 500,1500, and $2500 \mathrm{~K}$ for the Mach 10, 25, and 45 cases, respectively. The density of the freestream is varied to change the global Knudsen number of the flow as given in Table 1 . The Knudsen number is calculated using the sphere diameter as the characteristic length and the hard sphere model to calculate the mean free path. From Table 1 it is seen that the global Knudsen number varies from 0.002 to $\overline{0} .25$, giving a range of flow regimes from continuum to rarefied gas flow. The simulations are performed using two different computational methods, CFD and DSMC.

\section{Numerical Methods}

The DSMC simulations are performed using MONACO [11], a code developed at University of Michigan by Dietrich and Boyd. MONACO used DSMC to accurately simulate rarefied gas flows around hypersonic vehicles. MONACO is a parallel, unstructured, 2-D/3-D DSMC code, and it includes variable vibrational and rotational energy exchange models. MONACO can use either the variable hard sphere (VHS) or variable soft sphere collision models [2]; the VHS model is employed in this study. The final mesh used for each simulation is adapted by hand from previous simulations such that the cell size is of the same order as the mean free path. For this study a hybrid mesh is used with cell stretching. A hybrid mesh is one in which both a structured and unstructured grid is used. In this case a structured grid is employed along the fore body surface whereas an unstructured mesh is used everywhere else in the flowfield as shown in Fig. 1. For the structured grid, cell stretching is employed. This means the cell widths are adapted to be on the order of a mean free path whereas the cell heights are stretched larger than the mean free path. The amount of cell stretching varies depending on the global Knudsen number. The grid given in Fig. 1 has cells stretched by a factor of eight near the stagnation point. The amount of cell stretching decreases farther away from the stagnation point, and eventually the cells are no longer stretched. This procedure creates a larger cell volume so that more particles can populate the cells near the stagnation point. This is important in axisymmetric simulations for which it is difficult to obtain an appropriate number of particles per cell [12]. To obtain accurate results from a DSMC simulation, at least 20 particles per cell is suggested [2], and this is achieved in

Table 1 Simulated flow regimes

\begin{tabular}{lccc}
\hline \hline$K n_{\infty}$ & $\begin{array}{c}\text { Mass density, } \\
\mathrm{kg} / \mathrm{m}^{3}\end{array}$ & $\begin{array}{c}\text { Number density, } \\
\text { particles } / \mathrm{m}^{3}\end{array}$ & $\begin{array}{c}\text { Mean free } \\
\text { path, } \mathrm{m}\end{array}$ \\
\hline 0.002 & $9.875 \times 10^{-5}$ & $2.124 \times 10^{21}$ & $6.096 \times 10^{-4}$ \\
0.01 & $1.975 \times 10^{-5}$ & $4.247 \times 10^{20}$ & $3.048 \times 10^{-3}$ \\
0.05 & $3.949 \times 10^{-6}$ & $8.494 \times 10^{19}$ & $1.524 \times 10^{-2}$ \\
0.25 & $7.899 \times 10^{-7}$ & $1.699 \times 10^{19}$ & $7.62 \times 10^{-2}$ \\
\hline \hline
\end{tabular}




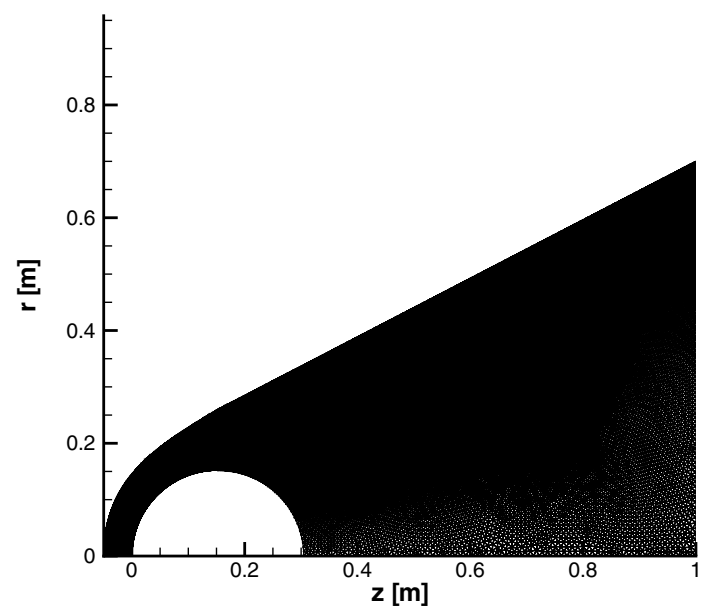

a) Full grid

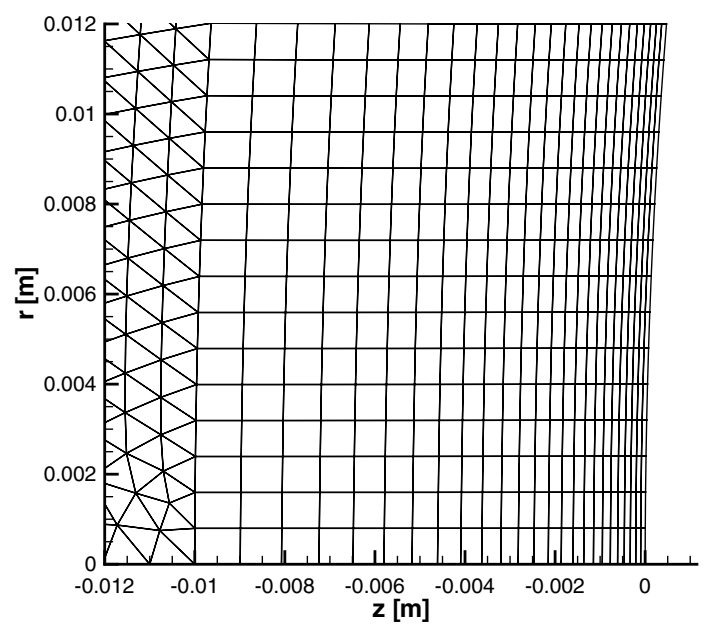

c) Stagnation point

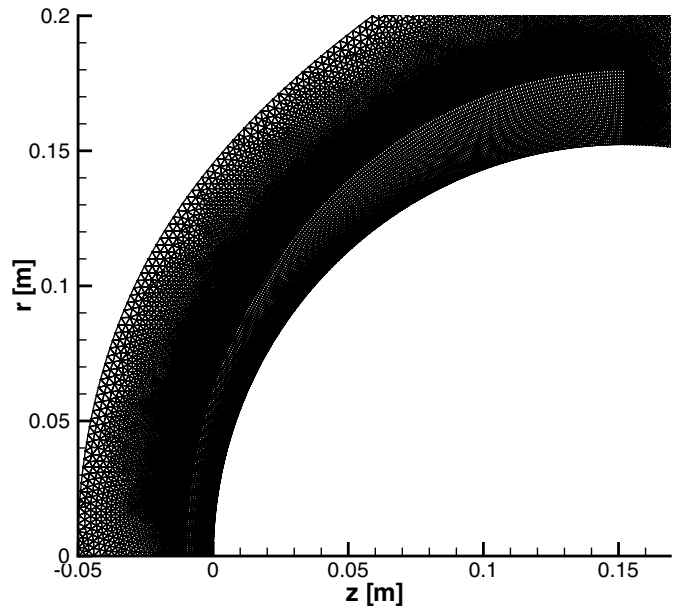

b) Fore body

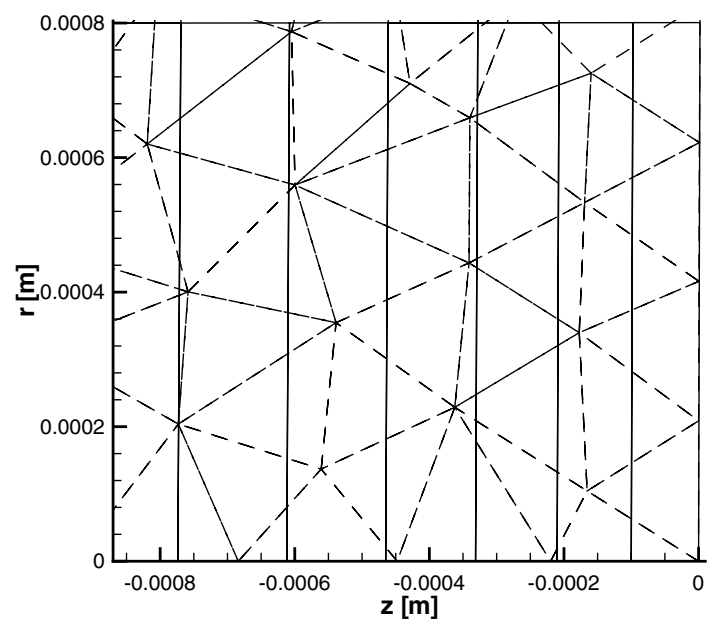

d) Hybrid and unstructured mesh

Fig. 1 The final tailored grid for the Mach 10 global Knudsen number 0.01 case for DSMC; all other DSMC grids are similar.

every simulation for this study. Cell stretching does not affect the simulation results because the primary flow gradients along the stagnation line are aligned with the cell widths, which are small enough to properly simulate the flow. Figure $1 \mathrm{~d}$ shows the unstructured mesh on top of a structured mesh near the stagnation point. From this figure it can be seen that the stretched cells give a much larger area than unstructured cells thus allowing more particles to populate the area near the stagnation point.

The CFD simulations are performed by solving the Navier-Stokes equations by use of the Michigan aerothermodynamic NavierStokes code LeMANS, developed at the University of Michigan for the simulation of hypersonic reacting flows $[13,14]$. LeMANS is a parallel, unstructured 2-D/3-D, finite-volume CFD code. LeMANS has the ability to simulate gases in chemical and thermal nonequilibrium. LeMANS employs a modified Steger-Warming flux vector splitting to discretize the numerical fluxes between cells, which has low dissipation and is appropriate near boundary layers. A point-implicit method is employed for the time march, but after a few hundred iterations is switched to a line-implicit method for faster convergence. Even though LeMANS can handle unstructured meshes, all the simulations performed for this study are carried out using structured meshes. It is necessary to ensure that the transport properties are the same in both CFD and DSMC; therefore, LeMANS is modified to use the same viscosity as the VHS method as given in Eqs. (4) and (5) [15]:

$$
\mu=\mu_{\text {ref }}\left(T / T_{\text {ref }}\right)^{\omega}
$$

$$
\mu_{\mathrm{ref}}=\frac{15 \sqrt{\pi m k T_{\mathrm{ref}}}}{2 \pi d_{\mathrm{ref}}^{2}(5-2 \omega)(7-2 \omega)}
$$

in which the variable hard sphere parameters are those used in the DSMC simulations and $\omega$ is 0.75 with a reference diameter and temperature of $4.17 \times 10^{-10} \mathrm{~m}$ and $273 \mathrm{~K}$, respectively.

\section{A. Rotational Relaxation}

Even though LeMANS is capable of modeling thermal nonequilibrium it does so with a two temperature model involving a coupled translational-rotational temperature and a coupled vibrational-electronic temperature. DSMC employs a variable rotational energy exchange probability [16] and therefore, simulates a separate rotational temperature. Because this study is concerned with comparisons between DSMC and CFD for simulations that involve significant amounts of nonequilibrium flow, it is important that the CFD code also has a separate rotational temperature to be able to match DSMC. Not only is it important to have rotational nonequilibrium for comparisons between CFD and DSMC, it is also beneficial to have a separate rotational energy equation that can provide more accurate results in hypersonic flows in which thermal nonequilibrium is common. The rotational energy equation per unit volume is given as follows:

$$
\frac{\partial E_{r}}{\partial t}+\nabla \cdot\left(E_{r} \mathbf{u}\right)=-\nabla \cdot\left(\mathbf{q}_{r}\right)-\nabla \cdot \sum_{s}\left(\rho_{s} e_{r s} \mathbf{u}_{d s}\right)+\dot{w}_{r}
$$


in which $q_{r}$ is the rotational heat flux given by Fourier's law, $u_{d s}$ is the species diffusion given by Fick's law, and $\dot{w}_{r}$ is a source term:

$$
\dot{w}_{r}=\sum_{s}\left(Q_{r s}^{t-r}+\dot{w}_{s} e_{r s}\right)
$$

Equation (7) is composed of two parts, the first being the rotational energy relaxation given in Eq. (8) and the second part is the gain and loss of rotational energy due to chemical reactions:

$$
Q_{r s}^{t-r}=\rho_{s} \frac{e_{r s}^{*}-e_{r s}}{Z_{r s} \tau_{c}}
$$

The rotational energy relaxation is modeled using a Landau-Teller model, in which $e_{r s}^{*}$ is the rotational energy evaluated at the translational temperature and $Z_{r s}$ and $\tau_{c}$ together give the rotational relaxation time. The rotational-collision number $Z_{r s}$ given in Eq. (9) derived by Parker [17] and $\tau_{c}$ is the mean collision time from the V $\overline{\mathrm{HS}}$ model. Equation (10) gives the mean collision rate, given by Bird [2], which is just the inverse of the mean collision time:

$$
\begin{aligned}
& Z_{r s}=Z_{r s}^{\infty} /\left[1+\frac{\pi^{\frac{3}{2}}}{2}\left(\frac{T_{s}^{*}}{T}\right)^{\frac{1}{2}}+\left(\frac{\pi^{2}}{4}+\pi\right)\left(\frac{T_{s}^{*}}{T}\right)\right] \\
& v_{s}=\sum_{i}\left(n_{i}\left(d_{\text {ref }}\right)_{i}^{2}\left(\frac{8 \pi k\left(T_{\text {ref }}\right)_{i}}{m_{i}^{*}}\right)^{\frac{1}{2}}\left(\frac{T}{\left(T_{\text {ref }}\right)_{i}}\right)^{1-\omega_{i}}\right)
\end{aligned}
$$

in which $Z_{r s}^{\infty}$ and $T^{*}$ are constants for a given species, and the VHS parameters are the same ones used in DSMC and the CFD viscosity model.

\section{B. Vibrational Relaxation}

The average probability of an inelastic collision in which vibrational energy is exchanged with translational energy is proportional to the inverse of the vibrational relaxation time. To approximate the vibrational relaxation time, a Landau-Teller model is used with correlated experimental data from Millikan and White [18]:

$$
\tau_{\mathrm{LT}}=\frac{1}{p} \exp \left[A\left(T^{-\frac{1}{3}}-B\right)-18.42\right]
$$

in which the pressure is in standard atmosphere, and the values of $A$ and $B$ come from Park [19] for both DSMC and CFD.

For high temperatures, which are often encountered in hypersonic flows, a correction proposed by Park [20] is employed as shown in Eq. (12). The total vibrational relaxation time is the sum of the Landau-Teller and Park relaxation times:

$$
\tau_{P}=1 / n \bar{c} \sigma
$$

in which $\sigma$ is the collision cross section, $\bar{c}$ is the mean speed, and $n$ is the number density.

A factor was proposed by Lumpkin et al. [21] to correct the relaxation time; although this was done for rotational relaxation, it can be applied to vibrational relaxation:

$$
\tau_{\text {part }}=\frac{\tau_{\text {cont }}}{1+\frac{\zeta}{4-2 \omega}}
$$

in which $\zeta$ is the vibrational degrees of freedom, $\tau_{\text {cont }}$ is the relaxation time from a continuum method, and $\tau_{\text {part }}$ is the relaxation time found in a particle method. The exchange of vibrational and translational energy in DSMC is handled by a phenomenological model as described by Boyd [22]. The average probability is used to find the instantaneous probabilities. Unfortunately, this process cannot be done analytically and so the method of steepest decent is employed.

When the instantaneous probability is integrated over all collisions it should match the average probability calculated from theory, but it was found that they do not. It is thought that the probabilities do not match due to the method of steepest descent required to find the instantaneous probability. It has been found that the probability can better correspond with theory by multiplying by a simple factor that is dependent on the maximum temperature [10]. Table $\underline{2}$ gives the probability from DSMC. It also gives the factor that should be used at a given maximum temperature. For this study the maximum temperature for the Mach 10 and 25 cases is approximately 5000 and $25,000 \mathrm{~K}$, respectively. The corresponding factor in Table 2 is used in the DSMC simulations. For the Mach 45 case the maximum temperature is approximately $90,000 \mathrm{~K}$, which is off the table. However, the factor tends to unity as the temperature climbs; a factor of 1 is used for the Mach 45 case.

\section{Slip Boundary Conditions}

At low Knudsen numbers, the no-slip boundary conditions hold. At higher Knudsen numbers, there are insufficient collisions near the wall and the flow is not able to equilibrate with the wall; hence, the no-slip condition is invalidated. In this study, the Gökçen and MacCormack [23] slip boundary conditions are used as given in Eq. (14):

$$
U_{s}=\left.A\left(\frac{2-\sigma}{\sigma}\right) \lambda \frac{\partial u_{x}}{\partial n}\right|_{n=0} \quad T_{0}-T_{w}=\left.\frac{2-\sigma}{\sigma} \lambda_{T} \frac{\partial T}{\partial n}\right|_{n=0}
$$

in which $U_{s}$ is the slip velocity, $T_{0}-T_{w}$ is the temperature jump, $\lambda$ is the mean free path, $A$ is a constant of proportionality taken as 1 in this study, and $\sigma$ is an accommodation coefficient given as follows:

$$
\sigma\left(a_{\lambda}-a_{w}\right)=\left.2 \lambda_{a} \frac{\partial a}{\partial n}\right|_{n=0}
$$

in which $a$ can be either velocity or temperature. For more information on slip boundary conditions in LeMANS please see Lofthouse [ㅁ].

\section{Results}

The purpose of this study is to compare surface properties predicted by DSMC and CFD simulations, heat flux, pressure, and shear stress to see if any differences occur due to continuum breakdown. Additionally, the integrated drag and the maximum heat flux are also compared from DSMC and CFD. The maximum heat flux predicted by DSMC is found by averaging the heat flux over the surface of the sphere within the first degree of the stagnation point. For CFD it will be seen that the maximum heat flux sometimes occurs slightly off the stagnation point. Because the CFD solutions are smooth there is no need for averaging and the maximum heat flux is found by finding the maximum value of the heat flux on the surface of the sphere.

The breakdown parameter is computed from both DSMC and CFD simulation results using Eq. (3), in which the derivative is taken in the direction of the maximum gradient. It is expected that continuum breakdown will occur in areas of high gradients, in the shock wave and boundary layer, and in areas of rarefied gas flow in the wake. It is also expected that the amount of continuum breakdown will increase with increasing Mach and Knudsen numbers. Also, a comparison of the present results to the cylinder case will be made to investigate any changes in continuum breakdown due to axisymmetry.

The results that are presented in this paper for the surface aerothermodynamic properties are given as nondimensionalized coefficients, which are defined as follows:

Table 2 Vibrational probabilities for $\mathbf{N}_{2}-\mathbf{N}_{2}$ collisions in DSMC compared with theory

\begin{tabular}{llll}
\hline \hline Temperature, K & DSMC & Theory & Factor \\
\hline 5,000 & 0.0002 & 0.0001 & 0.5 \\
10,000 & 0.0017 & 0.024 & 1.43 \\
25,000 & 0.009 & 0.0123 & 1.7 \\
30,000 & 0.0108 & 0.0172 & 1.59 \\
40,000 & 0.0138 & 0.0195 & 1.41 \\
50,000 & 0.0161 & 0.021 & 1.3 \\
\hline \hline
\end{tabular}




$$
\begin{gathered}
C_{p}=\frac{p-p_{\infty}}{\frac{1}{2} \rho_{\infty} U_{\infty}^{2}} \\
C_{\tau}=\frac{\tau}{\frac{1}{2} \rho_{\infty} U_{\infty}^{2}} \\
C_{q}=\frac{q}{\frac{1}{2} \rho_{\infty} U_{\infty}^{3}}
\end{gathered}
$$

in which $\infty$ indicates freestream conditions. The surface aerothermodynamic properties are plotted against the surface angle $\phi$, which is measured from the stagnation point.

\section{A. Rotational Nonequilibrium}

To test the validity of the rotational energy model, the temperature profiles from CFD and DSMC along the stagnation streamline are compared in Fig. 2 for several different flow conditions. Also included in these figures is the maximum gradient length local Knudsen number based on the DSMC simulation. This will give an idea of the amount of breakdown in the flow, but also gives a good approximate location of the shock. For the Mach 10 Knudsen number 0.002 case, the shock is very clearly defined by $K n_{\mathrm{GLL}}$, and it can be seen that the translational temperature, predicted by DSMC starts increasing further upstream due to the fact that the shock predicted by DSMC is thicker, as expected. However, the translational temperature from CFD quickly matches the temperature from DSMC although it does not capture the peak. It can be seen that there is rotational nonequilibrium behind the shock for both DSMC and CFD, but both rotational temperatures, nearly overlap each other along the stagnation streamline. For the Mach 10 Knudsen number 0.01 case, the shock is not as clearly defined and $K n_{\mathrm{GLL}}$ is greater than the 0.05 limit from the shock all the way to the wall indicating continuum breakdown has occurred. In this case, the difference in the shock thickness is much more noticeable. There is rotational nonequilibrium behind the shock for both CFD and DSMC, and again the difference in shock thickness leads to a difference in the rotational temperatures. However, the rotational temperature predicted by CFD catches and overshoots the DSMC rotational temperature post shock and eventually all temperatures equilibrate. This discrepancy can be explained by the fact that the flow is in nonequilibrium following the shock, which is verified by looking at velocity distribution functions at various points along the stagnation streamline. At the point at which the CFD temperatures overshoot the DSMC temperatures there is still a bimodal distribution indicating that the flow is in nonequilibrium.

Figures $2 \mathrm{c}$ and $2 \mathrm{~d}$ give the temperature profiles for Mach 25 and 45 at a global Knudsen number of 0.01 . At these higher Mach numbers, the simulations now include vibrational as well as rotational nonequilibrium. The Mach 25 case is similar to the Mach 10 case in that there is breakdown in the flow from the front of the shock all the way to the wall of the sphere. The difference in the shock thickness is again visible in the temperature profiles for DSMC and CFD. The vibrational temperature and the rotational temperature as predicted

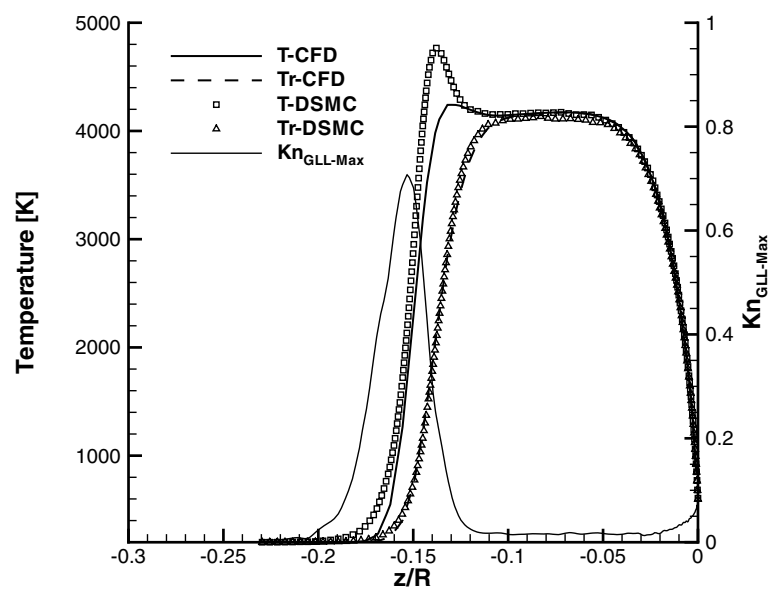

a) Mach $10, K n_{\infty}=0.002$

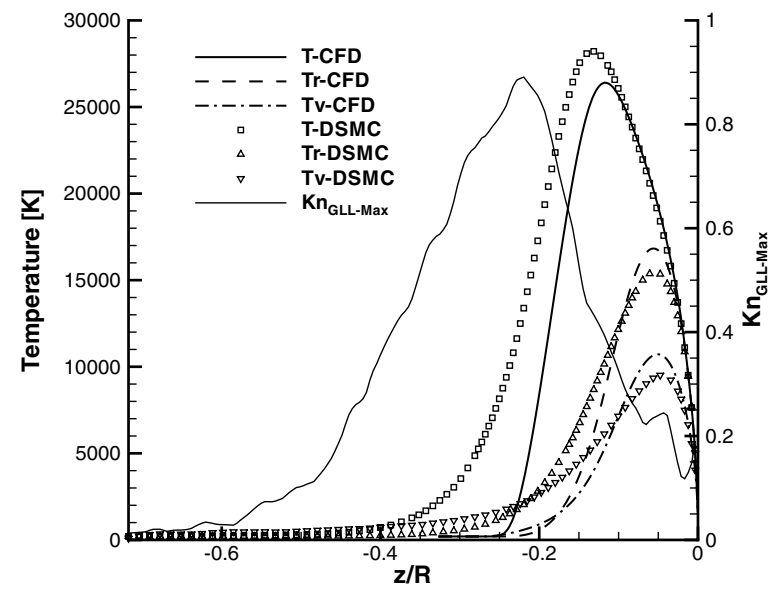

c) $\operatorname{Mach} 25, K n_{\infty}=0.01$

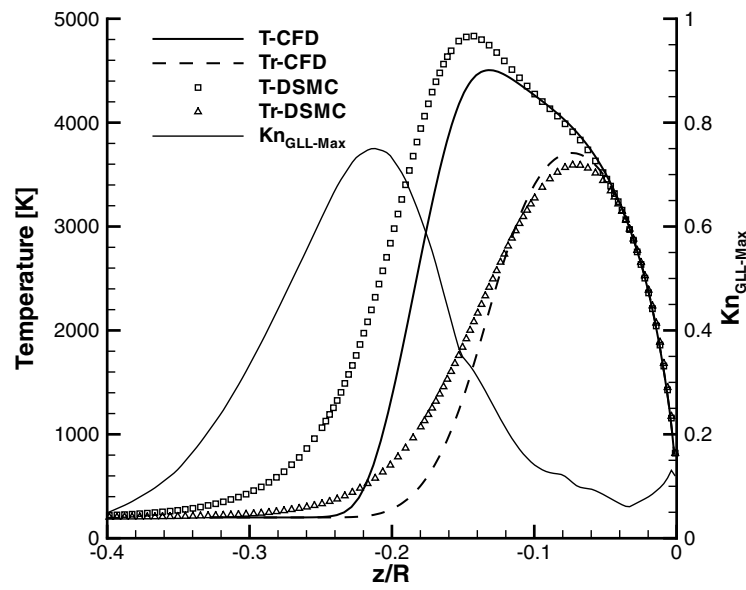

b) Mach 10, $K n_{\infty}=0.01$

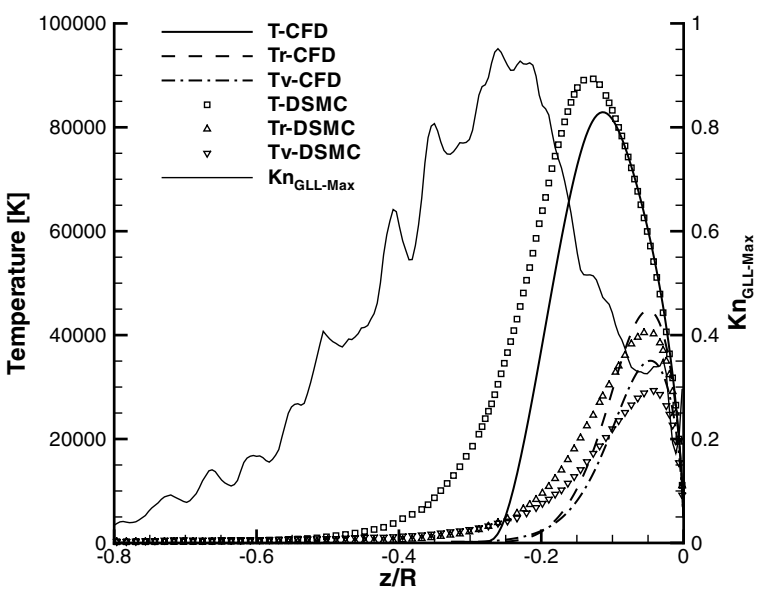

d) Mach 45, $K n_{\infty}=0.01$

Fig. 2 Temperature profiles along the stagnation line. The maximum $K n_{\mathrm{GLL}}$ is plotted on the right axis. Flow is from left to right; distance is normalized by the radius of the sphere. 
Table 3 Integrated drag, $\mathrm{N}$, (percent difference) from DSMC and CFD for Mach 10 flow

\begin{tabular}{lrcc}
\hline \hline$K n_{\infty}$ & DSMC & CFD, no slip & CFD, slip \\
\hline 0.002 & 29.02 & $28.93(-0.39 \%)$ & $28.91(-0.31 \%)$ \\
0.01 & 6.42 & $6.60(2.78 \%)$ & $6.47(0.79 \%)$ \\
0.05 & 1.60 & $1.83(14.04 \%)$ & $1.61(0.14 \%)$ \\
0.25 & 0.40 & $0.71(76.69 \%)$ & $0.43(6.39 \%)$ \\
\hline \hline
\end{tabular}

Table 4 Peak heating, $W / \mathrm{m}^{2}$, (percent difference) from DSMC and CFD for Mach 10 flow

\begin{tabular}{lccc}
\hline \hline$K n_{\infty}$ & DSMC & CFD, no slip & CFD, slip \\
\hline 0.002 & $1.09 \times 10^{5}$ & $1.15 \times 10^{5}(5.18 \%)$ & $1.12 \times 10^{5}(2.72 \%)$ \\
0.01 & $4.71 \times 10^{4}$ & $5.27 \times 10^{4}(11.89 \%)$ & $5.07 \times 10^{4}(7.68 \%)$ \\
0.05 & $2.11 \times 10^{4}$ & $2.54 \times 10^{4}(20.68 \%)$ & $2.28 \times 10^{4}(8.06 \%)$ \\
0.25 & $6.43 \times 10^{3}$ & $1.02 \times 10^{4}(58.23 \%)$ & $7.16 \times 10^{3}(11.42 \%)$ \\
\hline \hline
\end{tabular}

Table 5 Computational details for DSMC Mach 10 simulations

\begin{tabular}{lrrcc}
\hline \hline$K n_{\infty}$ & \multicolumn{1}{c}{ Cells } & \multicolumn{1}{c}{ Particles } & Time steps & CPU time, hours \\
\hline 0.002 & $1,293,365$ & $94,204,304$ & 250,000 & 12,240 \\
0.01 & 74,012 & $20,014,670$ & 143,000 & 4,608 \\
0.05 & 9,432 & $4,517,502$ & 157,000 & 384 \\
0.25 & 7,836 & $2,147,885$ & 139,000 & 192 \\
\hline \hline
\end{tabular}

Table 6 Computational details for CFD Mach 10 simulations

\begin{tabular}{lccc}
\hline \hline$K n_{\infty}$ & Cells & Iterations & CPU time, hours \\
\hline 0.002 & 48,000 & 20,000 & 320 \\
0.01 & 40,000 & 12,276 & 192 \\
0.05 & 26,000 & 14,000 & 112 \\
0.25 & 12,000 & 30,000 & 112 \\
\hline \hline
\end{tabular}

by CFD both overshoot their DSMC counterparts. However, all temperatures for both DSMC and CFD fall back into equilibrium at approximately the same point. For the Mach 45 case, the slight over prediction by CFD for all the temperatures still exists, but again all three temperatures reach equilibrium at the same point. Because the Mach number is so high in this case, the point at which equilibrium is reached is pushed close to the wall of the sphere.

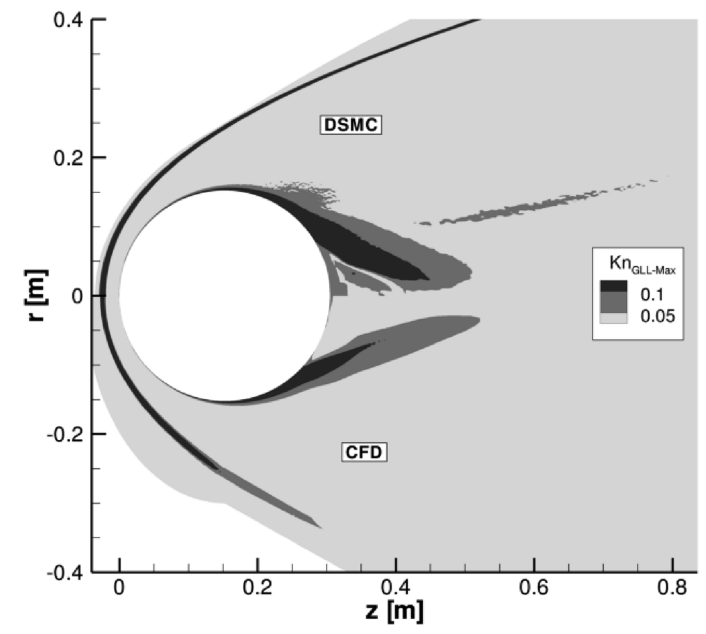

a) $K n_{G L L}$

\section{B. Effect of Global Knudsen Number}

To begin with, the results for the Mach 10 simulations at Knudsen numbers of $0.002-0.25$ are discussed in detail. The goal is to be able to discern the effects of changing the Knudsen number on continuum breakdown. The integrated drag and peak heat flux are given in Tables 3 and 4, respectively. The tables give the values predicted by DSMC and CFD along with a percent difference between the two. Because DSMC is a particle method that works in both the continuum regime and the rarefied regime it is assumed that the DSMC results are more accurate, so that the percent difference is calculated using the DSMC result as the correct result. At the lowest Knudsen number the integrated drag predictions are nearly identical and the peak heat flux values are close. However, as the Knudsen number grows the results diverge. It can be seen that slip boundary conditions improve the agreement between CFD and DSMC.

From the integrated drag and the total heat flux there is a correlation between increasing Knudsen number and increasing difference between DSMC and CFD results. Tables $\underline{5}$ and $\underline{6}$ give relevant computational details of the Mach 10 simulations from DSMC and CFD, respectively. The CFD cases in Table 6 are for simulations with no-slip boundary conditions. In general, simulations with slip boundary conditions took longer to converge. In the subsections to follow, the surface properties and $K n_{\mathrm{GLL}}$ are discussed in further detail.

\section{1. $K n_{\infty}=0.002$}

At a global Knudsen number of 0.002 , the flow is expected to be well within the continuum regime; therefore, CFD should have no problem properly simulating this flow. Even though the freestream conditions are in the continuum regime, there is still continuum breakdown in the flow, as shown in Fig. 3a. This figure shows that there is continuum breakdown in the shock and in the wake of the sphere. At this global Knudsen number, CFD and DSMC give approximately the same amount of continuum breakdown in the flow, even the shocks predicted by DSMC and CFD have the same thickness.

The surface coefficient of pressure is given in Fig. 3b. It can be seen that the pressure predicted by DSMC and CFD agree very well. A theoretical calculation of the coefficient of stagnation pressure, found in the inviscid limit to be 1.825 , compares well with the numerical prediction of 1.823 . The heat flux coefficient over the surface of the sphere is given in Fig. 4a. The CFD prediction of surface heat flux coefficient is higher than DSMC over most of the surface, but CFD under predicts DSMC over the back side of the sphere. An estimate of the stagnation point heat flux coefficient using a real gas Fay-Riddell analysis is found to be 0.0874 , which compares well to the numerically predicted value of 0.09 . The surface plots also show that $K n_{\text {GLL }}$ is greater than 0.05 over the whole surface, which means

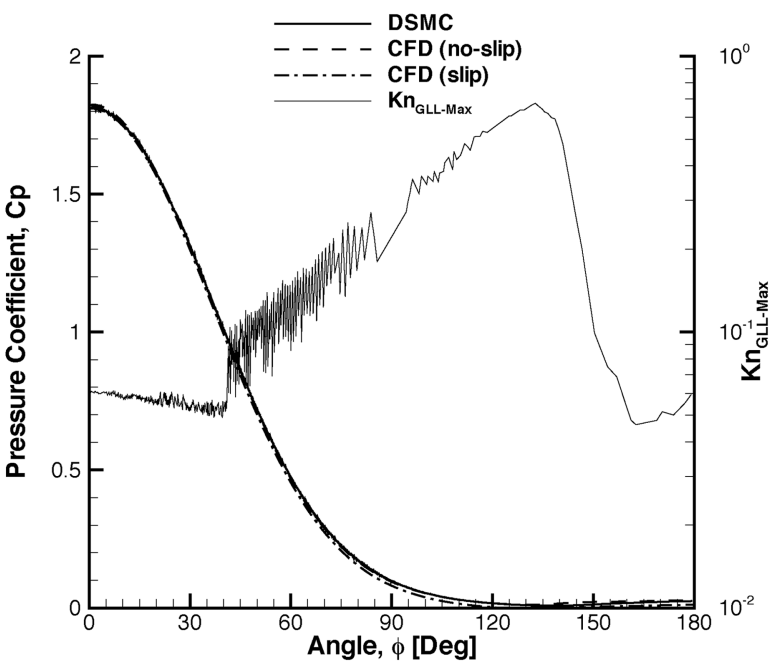

b) Coefficient of pressure

Fig. $3 K n_{\infty}=0.002: K n_{\text {GLL }}$ and surface pressure on a sphere in a Mach 10 flow of nitrogen. 


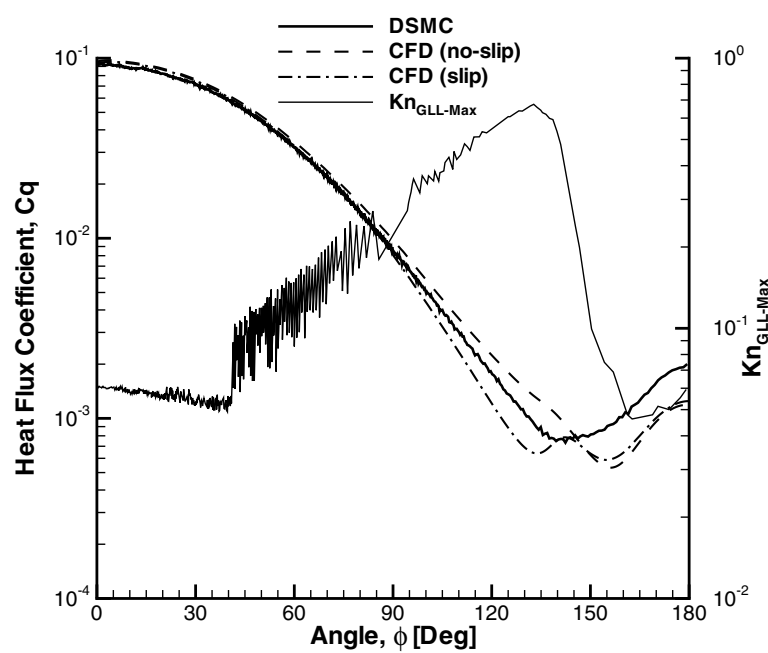

a) Coefficient of heat flux

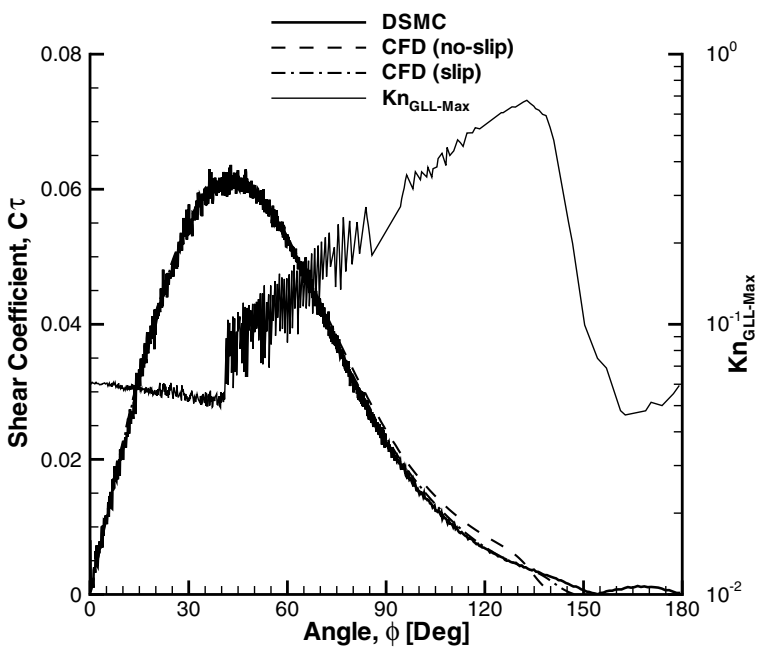

b) Coefficient of shear stress

Fig. $4 K n_{\infty}=0.002$ : surface heat flux and shear stress on a sphere in a Mach 10 flow of nitrogen.

breakdown has occurred, and this may explain the $5 \%$ difference in the peak heat transfer rate. The shear stress along the surface, as given in Fig. 4b, compares very well over most of the surface. The CFD and DSMC predictions for shear stress coefficient start to diverge over the backside of the sphere, which is to be expected because there is a large area of breakdown in the wake. From these figures it is seen that slip boundary conditions have little effect on the surface properties. This confirms what is already known: at low Knudsen numbers no slip is a good approximation.

\section{2. $K n_{\infty}=0.01$}

The traditional, but often debated, limit for accurate CFD simulations is a global Knudsen number of 0.01. From Fig. 5a it can be seen from the contours of $K n_{\mathrm{GLL}}$ that there is breakdown occurring in the shock, near the wall and in the wake of the sphere. It should be noted that at this global Knudsen number, DSMC predicts a larger amount of breakdown in the wake than CFD. Also, at this condition, the shock is noticeably thicker in the DSMC case whereas the location of the shock is approximately the same for DSMC and CFD.

Even at this higher global Knudsen number, the pressures from DSMC and CFD still agree very well. For this case the flow is in continuum breakdown from the shock all the way to the wall on the stagnation streamline, which may explain the small discrepancies in pressure. The surface heat flux coefficient predicted by CFD with no slip is always larger than DSMC, however, slip boundary conditions significantly improve the heat flux predicted by CFD especially on the aft body as shown in Fig. 6a. The shear stress coefficient, given in Fig. $6 \mathrm{~b}$, compares well between the two techniques near the stagnation region but then begins to diverge over the latter part of the surface. The slip boundary condition improves the agreement between CFD and DSMC for the shear stress over the aft body. For this case it is easy to notice that the shear and heat flux on the surface are higher in CFD than DSMC, but the slip boundary condition improves the agreement between CFD and DSMC. The surface profile of $K n_{\mathrm{GLL}}$ shows that the entire surface is considered to be in breakdown, which may explain the disagreement between CFD and DSMC.

\section{3. $K n_{\infty}=0.05$}

At a global Knudsen number of 0.05 , the flow is outside the supposed limit for physically accurate CFD simulations; it is in the transition regime between continuum flow and a rarefied gas. The flow does show that there is a large amount of breakdown in the shock, boundary layer, and wake, as seen in Fig. 7a. From this figure it is seen that $K n_{\mathrm{GLL}}$ exceeds the critical value of $\overline{0.05}$ for a large part of the domain for both CFD and DSMC. This means that continuum breakdown has occurred and CFD should have difficulty simulating the flow accurately. Although the amount of breakdown in the shock is approximately the same, it can be seen that the breakdown predicted by CFD is larger in the wake.

The surface pressure on the sphere simulated by CFD and DSMC is still in very good agreement as shown in Fig. $7 \mathrm{~b}$. One oddity is that

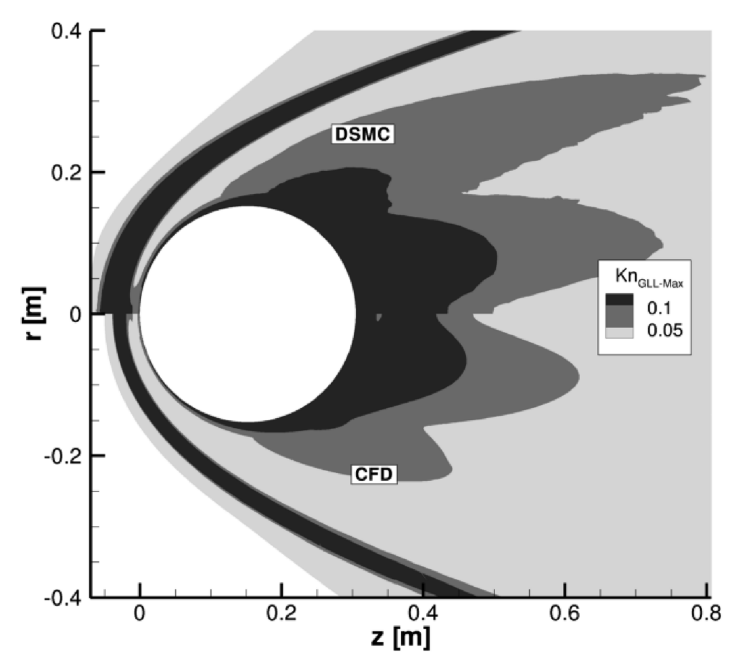

a) $K n_{G L L}$

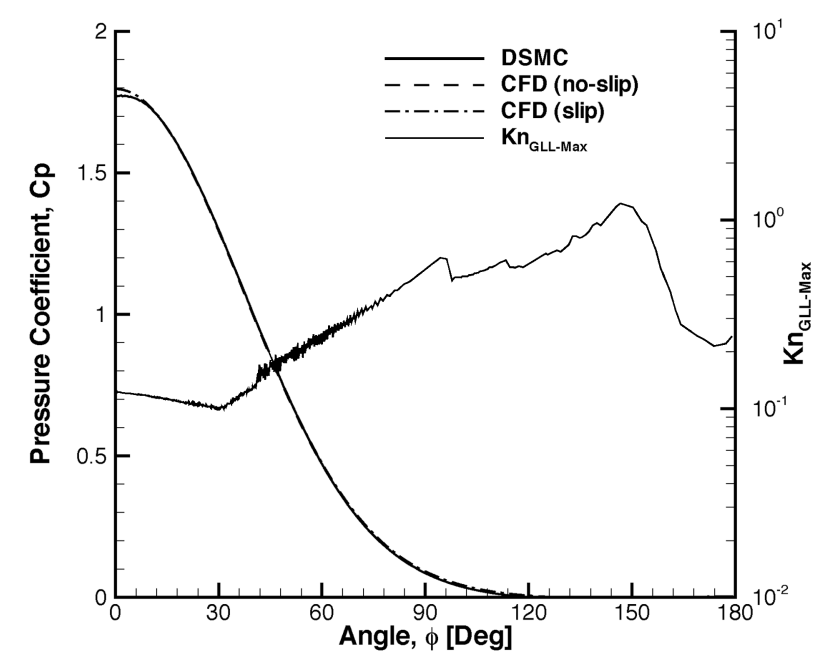

b) Coefficient of pressure

Fig. $5 K n_{\infty}=0.01: K n_{G L L}$ and surface pressure on a sphere in a Mach 10 flow of nitrogen. 


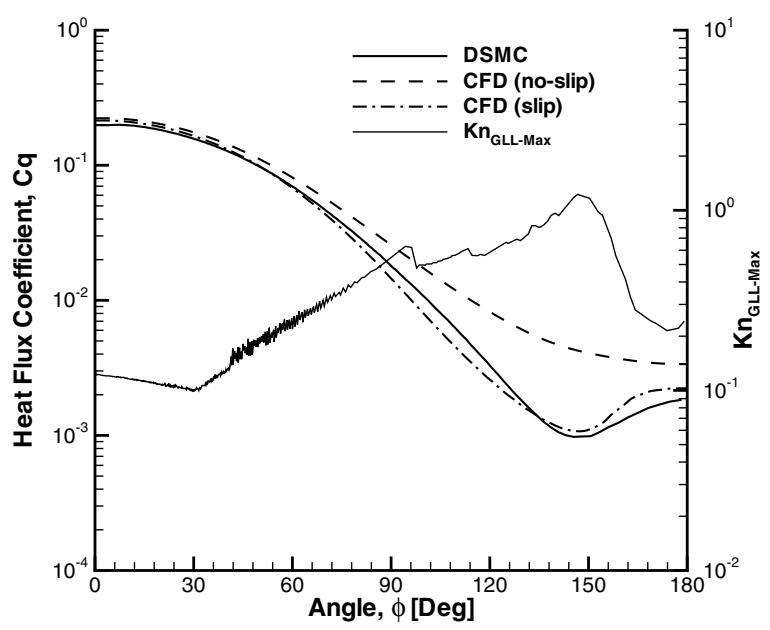

a) Coefficient of heat flux

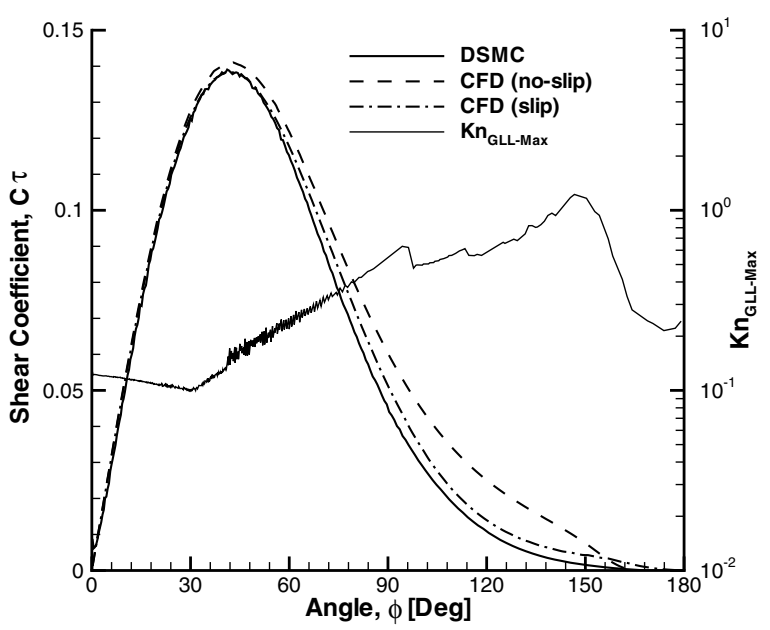

b) Coefficient of shear stress

Fig. $6 K n_{\infty}=0.01$ : surface heat flux and shear stress on a sphere in a Mach 10 flow of nitrogen.

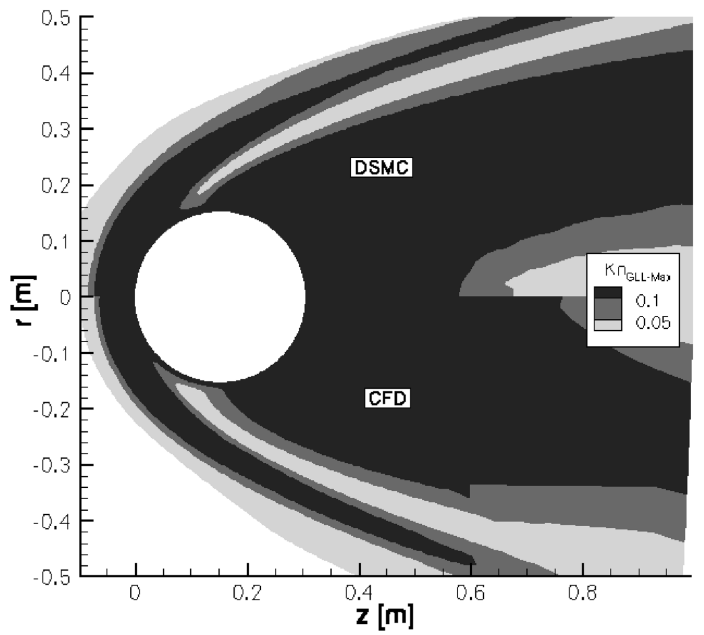

a) $K n_{G L L}$

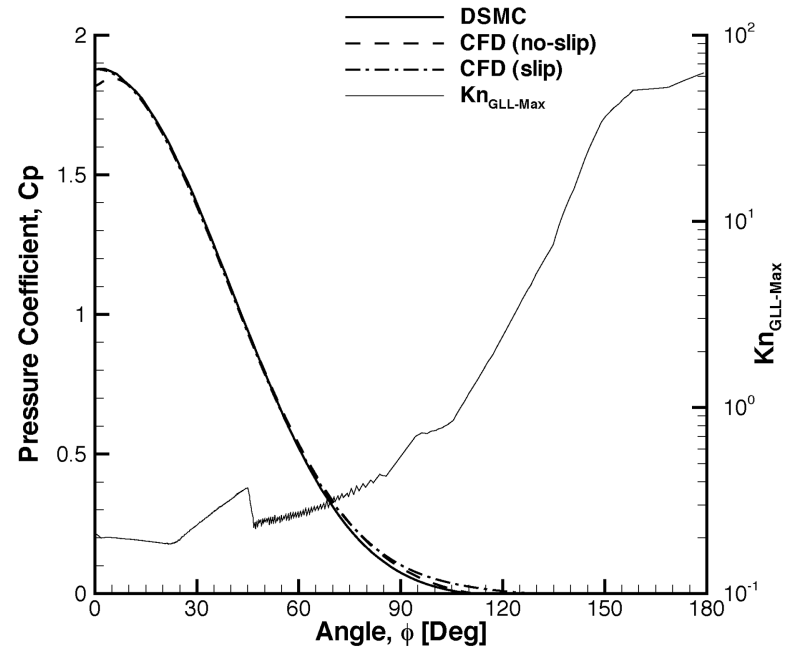

b) Coefficient of pressure

Fig. $7 K n_{\infty}=0.05: K n_{\mathrm{GLL}}$ and surface pressure on a sphere in a Mach 10 flow of nitrogen.

the CFD prediction of pressure is less than DSMC at the stagnation point, although this effect is not observed with slip boundary conditions. The surface heat flux, as seen in Fig. 8a, displays a large separation between CFD with no slip and DSMC. This separation starts at the stagnation point and goes all the way to the backside of the sphere. When the slip boundary condition is used the heat transfer coefficient drops, improving the agreement between CFD and DSMC, although not as much as the peak heating comparison would

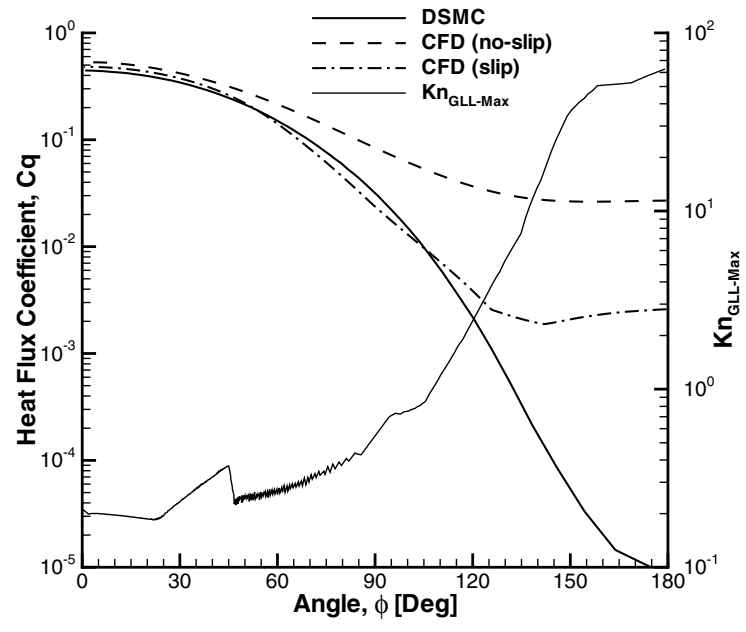

a) Coefficient of heat flux

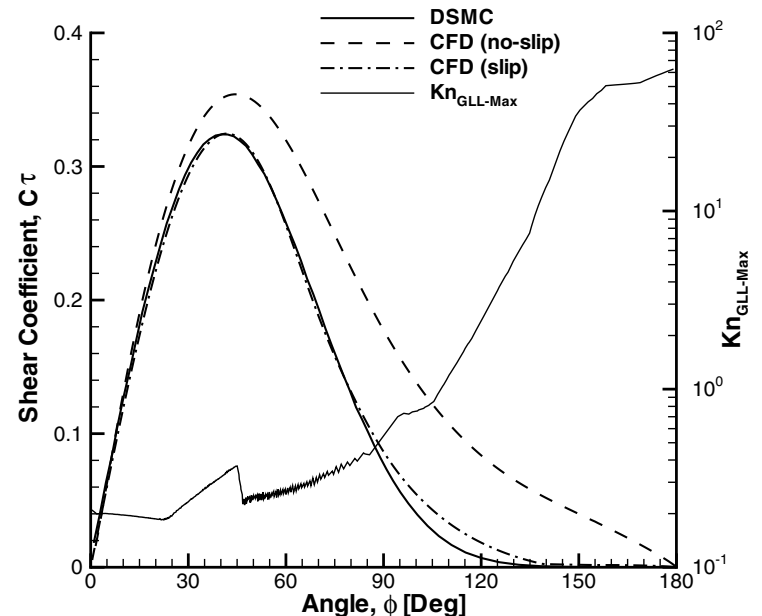

b) Coefficient of shear stress

Fig. $8 K n_{\infty}=0.05$ : surface heat flux and shear stress on a sphere in a Mach 10 flow of nitrogen. 


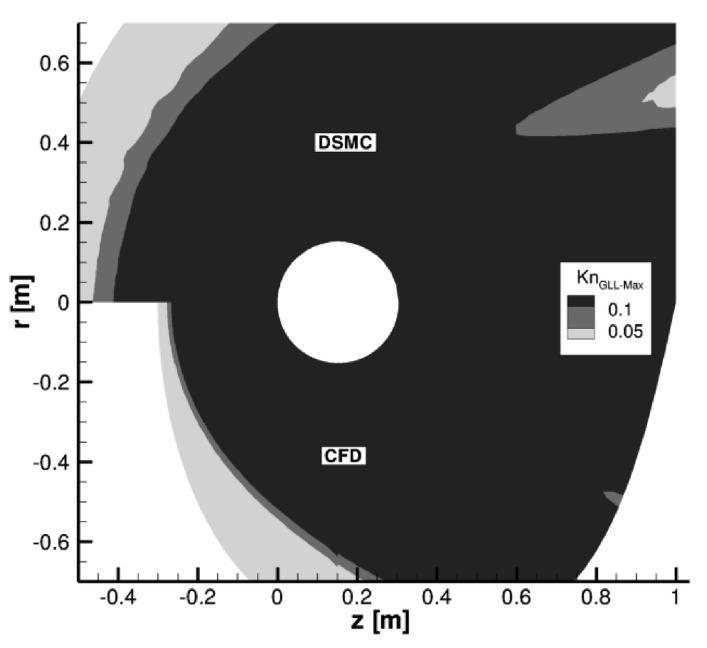

a) $K n_{G L L}$

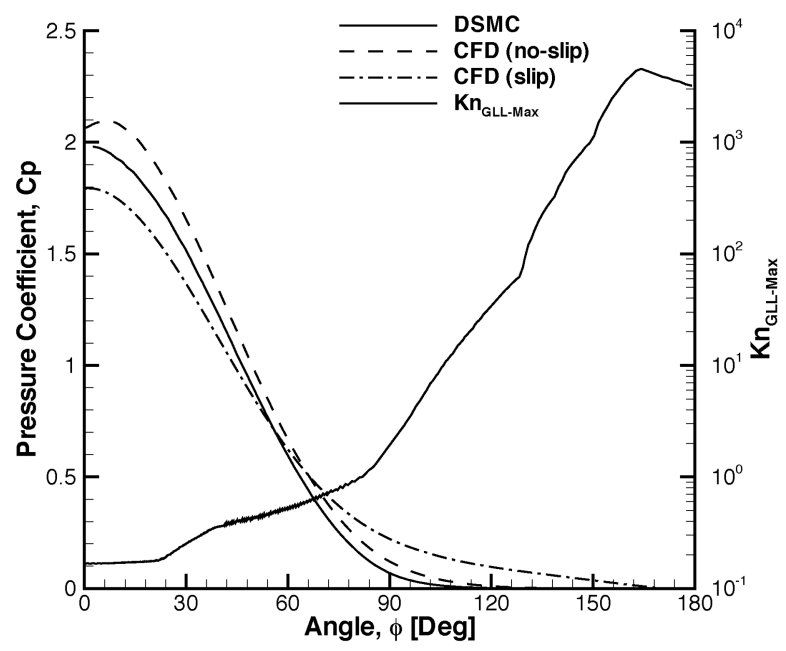

b) Coefficient of pressure

Fig. $9 K n_{\infty}=0.25: K n_{\text {GLL }}$ and surface pressure on a sphere in a Mach 10 flow of nitrogen.

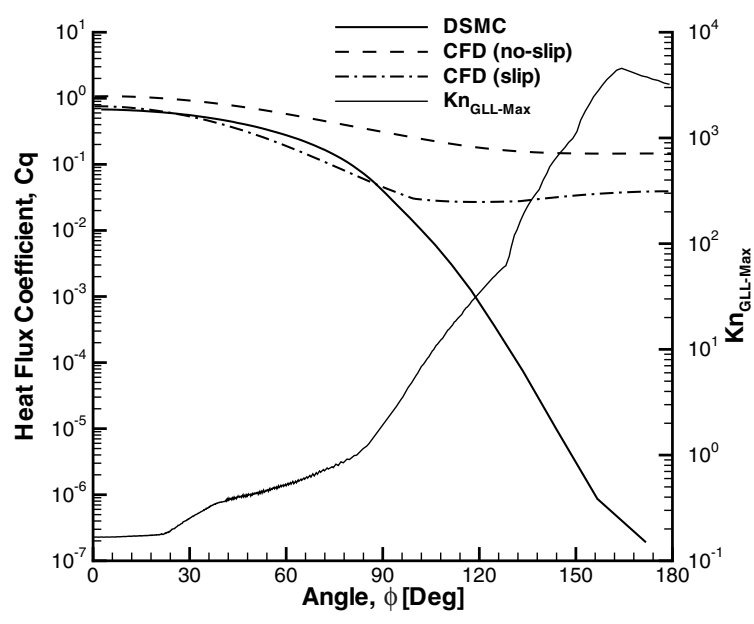

a) Coefficient of heat flux

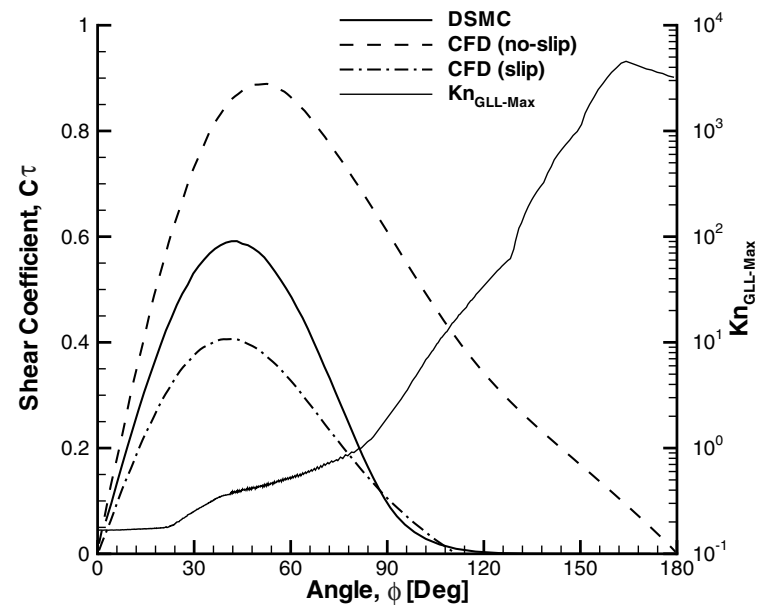

b) Coefficient of shear stress

Fig. $10 K n_{\infty}=0.25$ : surface heat flux and shear stress on a sphere in a Mach 10 flow of nitrogen.

lead one to believe. The shear stress, given in Fig. 8b, for both DSMC and CFD with no slip agree very well over the first $25 \mathrm{deg}$, but then the two diverge over the rest of the surface due to the growing amount of breakdown. The CFD with no-slip prediction for the surface shear stress gives the peak value at a slightly later point on the surface and gives a value higher than DSMC does. The slip boundary condition significantly improves the agreement between CFD and DSMC for the shear stress, giving the same peak value at the same location as DSMC.

\section{4. $K n_{\infty}=0.25$}

The last and highest global Knudsen number that is discussed in this work is 0.25 . This gives a flow that is well outside of the continuum regime and is now a rarefied gas. At this Knudsen number, the flow is nearly entirely in continuum breakdown, as seen in Fig. 9a. There is only a small portion that is not in continuum breakdown behind the sphere for DSMC. At this global Knudsen

Table 7 Integrated drag, $\mathbf{N}$, (percent difference) for DSMC and CFD for a Knudsen number of 0.01

\begin{tabular}{lccc}
\hline \hline Mach number & DSMC & CFD, no slip & CFD, slip \\
\hline 10 & 6.42 & $6.60(2.78 \%)$ & $6.47(0.79 \%)$ \\
25 & 41.2 & $42.44(2.99 \%)$ & $40.34(-2.09 \%)$ \\
45 & 134.85 & $141.39(4.85 \%)$ & $133.17(-1.25 \%)$ \\
\hline \hline
\end{tabular}

number, the shock is very far out in front of the sphere, approximately $0.4 \mathrm{~m}$ from the stagnation point, whereas the other cases are all less than $0.1 \mathrm{~m}$ from the stagnation point.

At a global Knudsen number of 0.25 , the flow is rarefied and as a result the entire flow is considered to be in continuum breakdown, which has a significant effect on the surface properties of the sphere. Figure $9 \mathrm{~b}$ gives the coefficient of pressure along the surface of the sphere. It is interesting to note that at this global Knudsen number, the no-slip CFD predicted coefficient of pressure goes above 2 at the stagnation point. A theoretical calculation of the coefficient of stagnation pressure, found in the free-molecular limit [24] to be 2.13, shows that a value near two is still within reason at this high a global Knudsen number. The surface heat flux coefficient, given in Fig. 10a, shows that there is a large difference between DSMC and CFD with no slip over the whole surface of the sphere. An estimate of the stagnation point heat flux coefficient in the free-molecular limit is found to be 0.941 verifying that the numerically found value of 0.7 is within reason. Figure $10 \mathrm{~b}$ shows the shear stress coefficient along the

Table 8 Peak heating, $\mathrm{W} / \mathrm{m}^{2}$, (percent difference) for DSMC and CFD for a Knudsen number of 0.01

\begin{tabular}{lccc}
\hline \hline Mach number & DSMC & CFD, no slip & CFD, slip \\
\hline 10 & $4.71 \times 10^{4}$ & $5.27 \times 10^{4}(11.89 \%)$ & $5.07 \times 10^{4}(7.68 \%)$ \\
25 & $9.81 \times 10^{5}$ & $1.08 \times 10^{6}(10.22 \%)$ & $1.03 \times 10^{6}(4.87 \%)$ \\
45 & $6.84 \times 10^{6}$ & $7.55 \times 10^{6}(10.47 \%)$ & $7.14 \times 10^{6}(4.41 \%)$ \\
\hline \hline
\end{tabular}


Table 9 Computational details for DSMC Kn$n_{\infty}=\mathbf{0 . 0 1}$ simulations

\begin{tabular}{lrccc}
\hline \hline Mach & \multicolumn{1}{c}{ Cells } & Particles & Time steps & CPU time, hours \\
\hline 10 & 74,012 & $20,014,670$ & 143,000 & 4,608 \\
25 & 103,985 & $14,213,372$ & 149,000 & 1,152 \\
45 & 119,959 & $71,345,469$ & 150,000 & 4,608 \\
\hline \hline
\end{tabular}

Table 10 Computational details for CFD $K n_{\infty}=0.01$ simulations

\begin{tabular}{lccc}
\hline \hline$K n_{\infty}$ & Cells & Iterations & CPU time, hours \\
\hline 10 & 40,000 & 12,276 & 192 \\
25 & 40,000 & 16,358 & 192 \\
45 & 40,000 & 28,806 & 384 \\
\hline \hline
\end{tabular}

surface of the sphere. The shear stresses agree well near the stagnation point, but then DSMC and CFD start to diverge. The shear stress predicted by DSMC gives a peak at a smaller angle and at a much smaller magnitude than CFD with no slip. Using the slip boundary conditions in this case did not improve the agreement, this is not surprising given the global Knudsen number is in the rarefied regime and CFD with the slip boundary condition is not expected to perform well.

\section{Effect of Mach Number}

The next set of results discussed is from simulations at a global Knudsen number of 0.01 for Mach numbers of 10, 25, and 45 . This portion of the study aims to discern the effects of changing the Mach number on continuum breakdown, while freezing the global Knudsen number. Because the Mach 10 simulation was discussed as part of the results in the last section it will not be discussed in this section. Therefore, this section begins by discussing the results of Mach 25 at a global Knudsen number of 0.01 . The integrated drag and peak heat flux are given in Tables $\underline{7}$ and 8 , respectively. The tables give the values predicted by DSMC $\overline{\text { and }} \overline{\mathrm{CFD}}$ along with a percent difference between DSMC and CFD. Even at Mach 10 there is a sizable difference between CFD and DSMC for the integrated drag and total heat flux. It is interesting to note that the differences do not increase with increasing Mach number. The slip boundary condition improves the agreement between CFD and DSMC across all Mach numbers for integrated drag and peak heat flux.

From the integrated drag and the total heat flux there does not appear to be a correlation between Mach number and the percent differences between DSMC and CFD results. However, this does not mean that Mach number has not affected continuum breakdown. In the subsections to follow, the surface properties and $K n_{\mathrm{GLL}}$ are discussed in further detail. Pertinent computational details for the global Knudsen number of 0.01 simulations are given in Tables 9 and 10 for DSMC and CFD, respectively. The CFD details given in Table 10 are for simulations with no-slip boundary conditions.

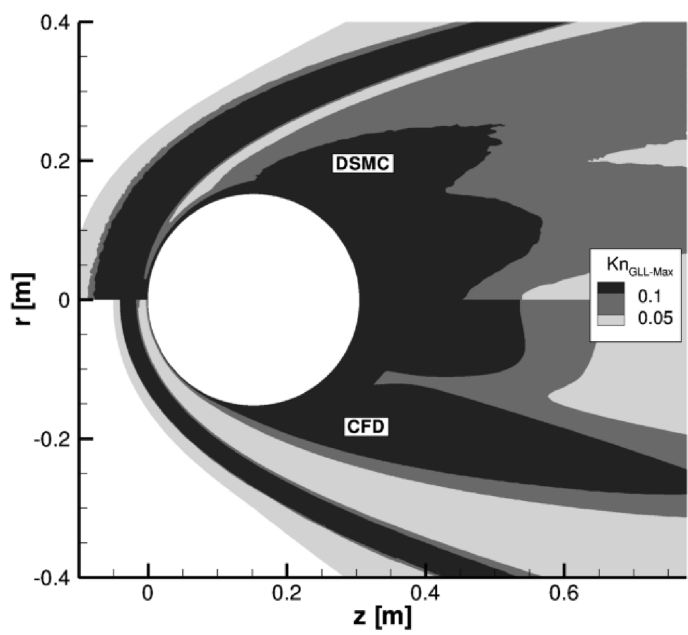

a) $K n_{G L L}$

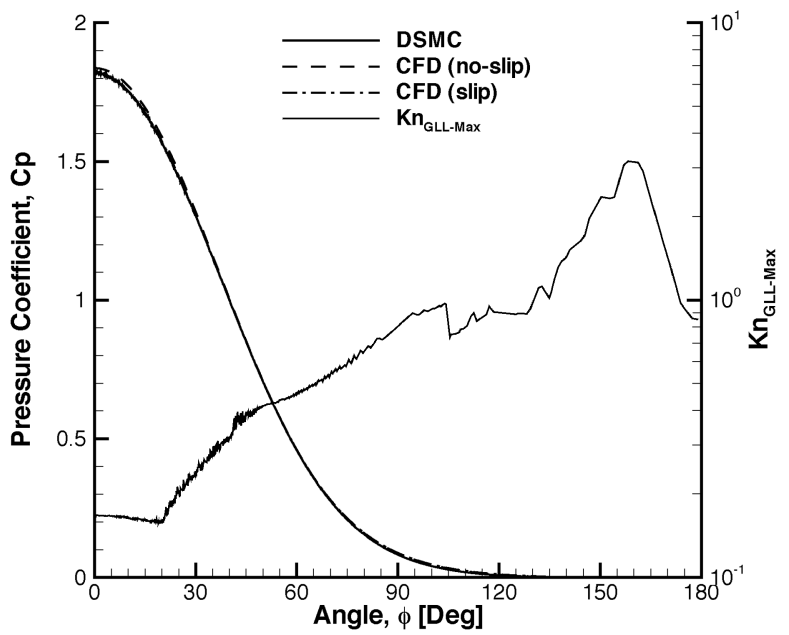

b) Coefficient of pressure

Fig. $11 K n_{\infty}=0.01: K n_{\text {GLL }}$ and surface pressure on a sphere in a Mach 25 flow of nitrogen.

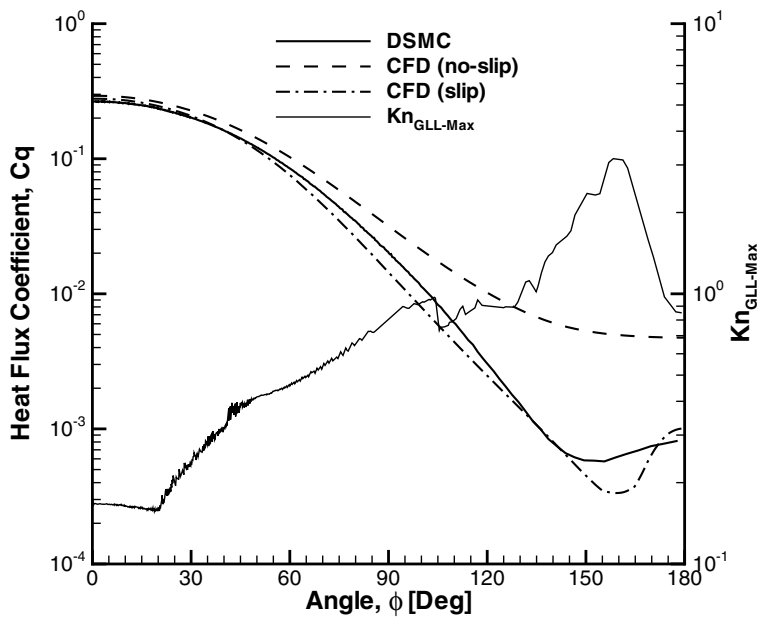

a) Coefficient of heat flux

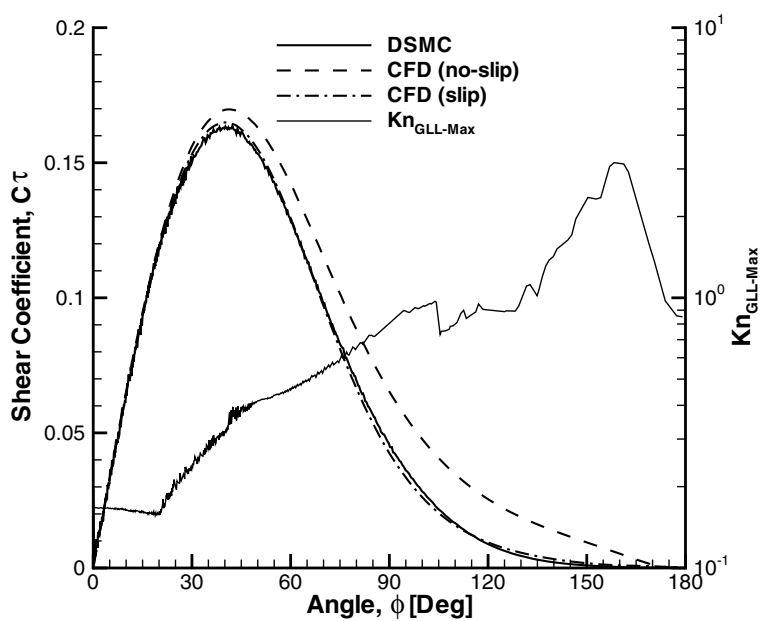

b) Coefficient of shear stress

Fig. $12 K n_{\infty}=0.01$ : surface heat flux and shear stress on a sphere in a Mach 25 flow of nitrogen. 


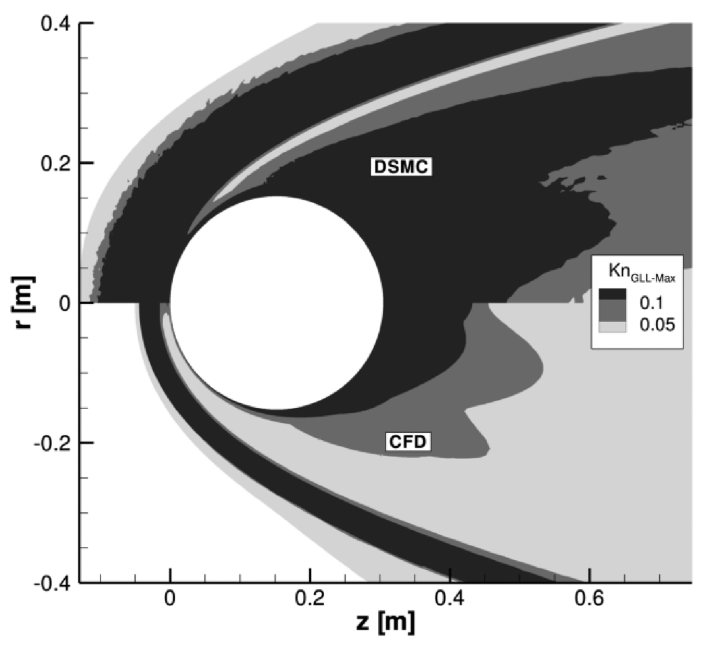

a) $K n_{G L L}$

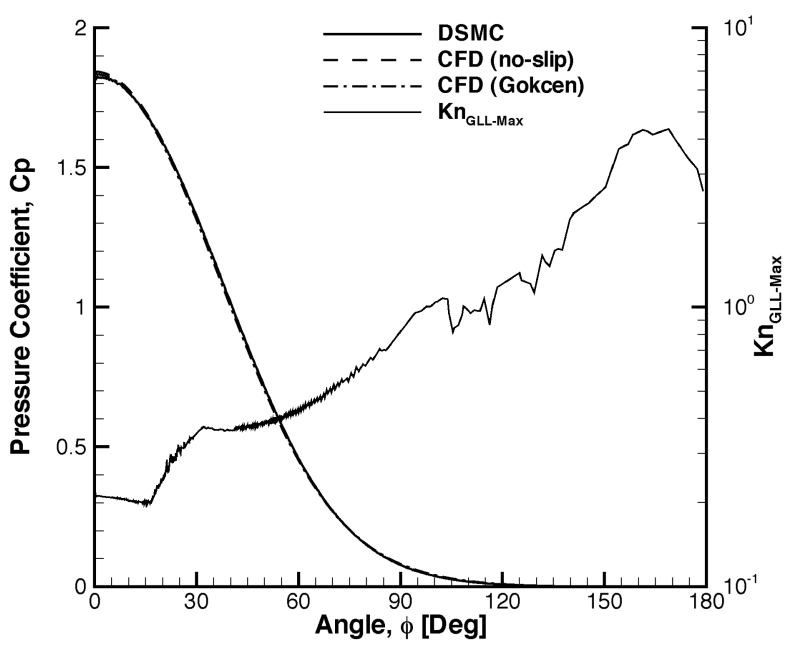

b) Coefficient of pressure

Fig. $13 K n_{\infty}=0.01: K n_{\mathrm{GLL}}$ and surface pressure on a sphere in a Mach 45 flow of nitrogen.

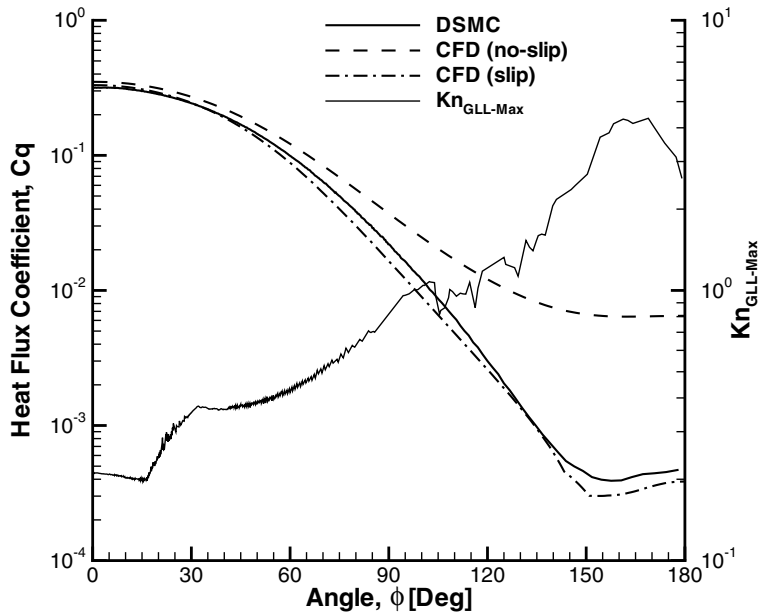

a) Coefficient of heat flux

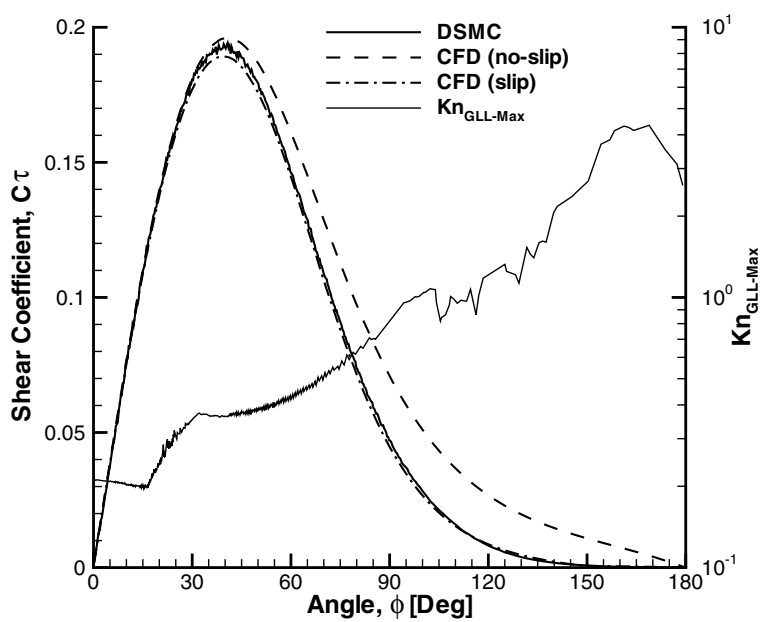

b) Coefficient of shear stress

Fig. $14 K n_{\infty}=0.01$ : surface heat flux and shear stress on a sphere in a Mach 45 flow of nitrogen.

\section{Mach 25}

At this Mach number, there is rotational and vibrational nonequilibrium. From Fig. 11a it is seen that there is breakdown occurring in the shock, near the wall and in the wake of the sphere. It should be noted that at this Mach number, DSMC predicts a larger amount of breakdown in the wake, and there is breakdown in the flow

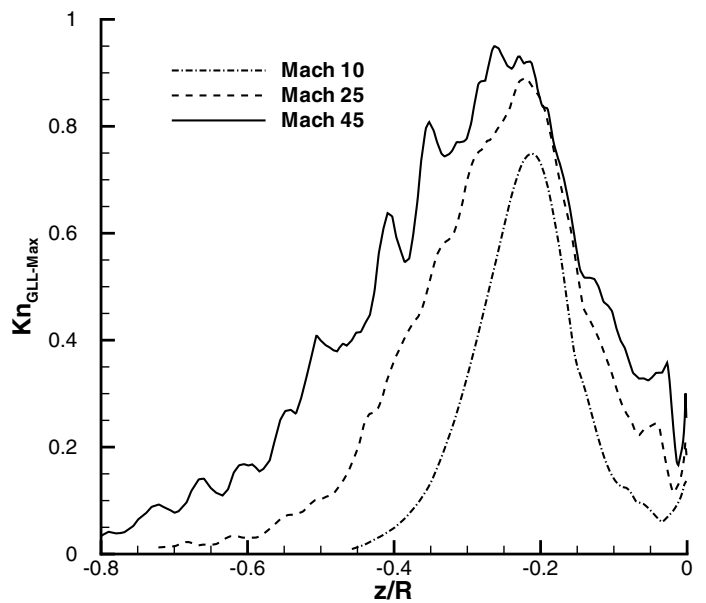

Fig. $15 K n_{\text {GLL }}$ along the stagnation streamline at a global Knudsen number of 0.01 . from the shock all the way to the wall along the stagnation streamline. Also, the amount of continuum breakdown predicted by DSMC is greater than the amount of breakdown predicted by CFD.

Even at a higher Mach number, the pressures from DSMC and CFD still agree very well, as seen in Fig. 11b. The surface heat flux coefficient, given in Fig. 12a, predicted by no-slip CFD is always larger than DSMC. However, the slip boundary condition significantly improves the agreement between CFD and DSMC for the heat transfer coefficient. The shear stress coefficient, given in Fig. 12b, compares well between the two techniques near the stagnation region but then begins to diverge over the latter part of the sphere. From the plot of the shear stress coefficient it is seen that although DSMC has a lower magnitude at the peak, the peak for both DSMC and CFD occurs at approximately the same location. Again, the slip boundary condition improves the agreement between CFD and DSMC for the shear stress coefficient. The results for this case are very similar to the Mach 10 case, even though the Mach number more than doubled.

\section{Mach 45}

At this Mach number, there is breakdown in the shock and the wake of the flow as seen in Fig. 13a, and again the flow from the shock all the way to the wall along the stagnation streamline is in continuum breakdown. From the figure it is seen that the amount of breakdown predicted by DSMC is larger in the shock region and in the wake than predicted by CFD. 
Table 11 Integrated drag, N, N/mfor cylinder, for DSMC and CFD for a Knudsen number of 0.01 and a Mach number of 10

\begin{tabular}{lrrc}
\hline \hline & DSMC & CFD & Percent difference \\
\hline Cylinder & 33.98 & 33.75 & -0.66 \\
Sphere & 6.42 & 6.60 & 2.78 \\
\hline \hline
\end{tabular}

Table 12 Peak heating, $\mathrm{W} / \mathrm{m}^{2}$ for DSMC and CFD for a Knudsen number of 0.01 and a Mach number of 10

\begin{tabular}{lccc}
\hline \hline & DSMC & CFD & Percent difference \\
\hline Cylinder & $3.2 \times 10^{4}$ & $3.23 \times 10^{4}$ & 1.12 \\
Sphere & $4.71 \times 10^{4}$ & $5.27 \times 10^{4}$ & 11.89 \\
\hline \hline
\end{tabular}

The surface pressure coefficient, given in Fig. 13b, for DSMC and CFD match very well over the surface of the sphere. The surface heat flux coefficient displays a gap between CFD with no slip and DSMC over the entire surface of the sphere, as shown in Fig. 14a. However, the use of slip boundary condition improves the heat flux predicted by CFD and now matches much more closely with what was predicted by DSMC. The surface shear stress, as given in Fig. 14b, for CFD and DSMC agree well over the beginning of the sphere. The

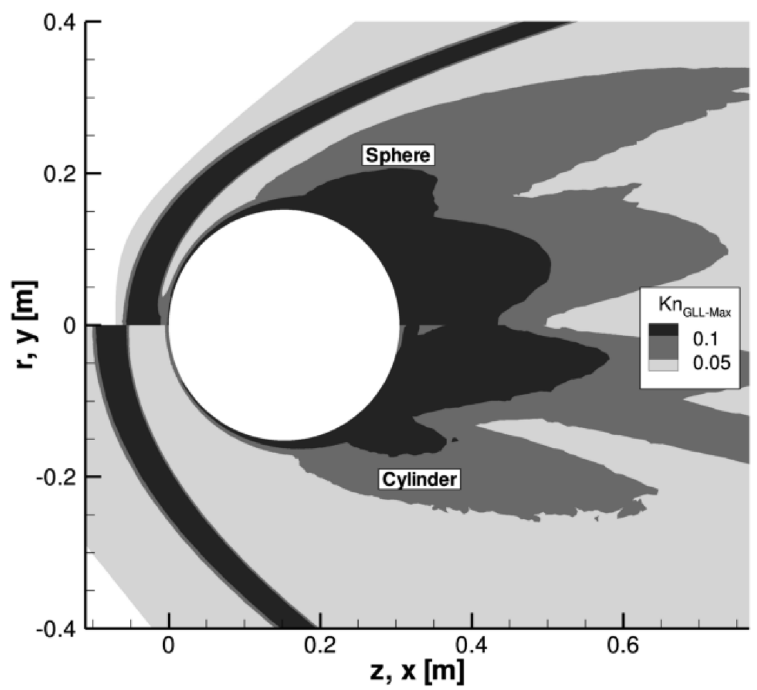

a) DSMC
CFD shear stress has a higher magnitude at the peak, but both methods predict the peak at nearly the same location. After the peak, the CFD and DSMC simulations of the shear stress coefficient diverge. The slip boundary condition shows an improved agreement between CFD and DSMC for shear stress coefficient.

Because there is no clear trend with increasing Mach number, as there is with increasing global Knudsen number, one can conclude that Mach number does not affect the surface properties in the same way as Knudsen number. Even though the amount of continuum breakdown has increased with increasing Mach number, the difference in the surface properties between CFD and DSMC remains relatively constant. This behavior can be better understood by looking at $K n_{\mathrm{GLL}}$ along the stagnation streamline for the three Mach numbers, as given in Fig. 15. From this figure it is seen that the region of continuum breakdown increases with Mach number, but the variation of $K n_{\mathrm{GLL}}$ is not significantly changed. Continuum breakdown increases because the thickness of the shock increases, that is seen in the figure by the fact that the profile of $K n_{\mathrm{GLL}}$ widens with increasing Mach number.

\section{Comparison of Sphere and Cylinder Flows}

A further part of this study compares the results of the sphere with that of the analogous cylinder case. This is performed to compare the amount of continuum breakdown and the effects on the surface properties caused by changing the geometry. Because the main focus

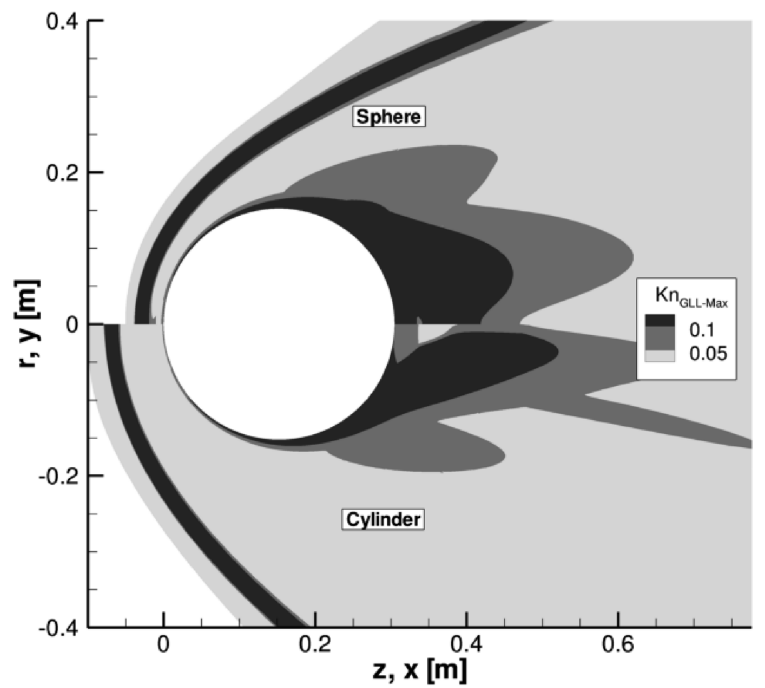

b) CFD

Fig. $16 K n_{\infty}=0.01: K n_{\text {GLL }}$ from DSMC and CFD in a Mach 10 flow of nitrogen over a sphere and a cylinder.

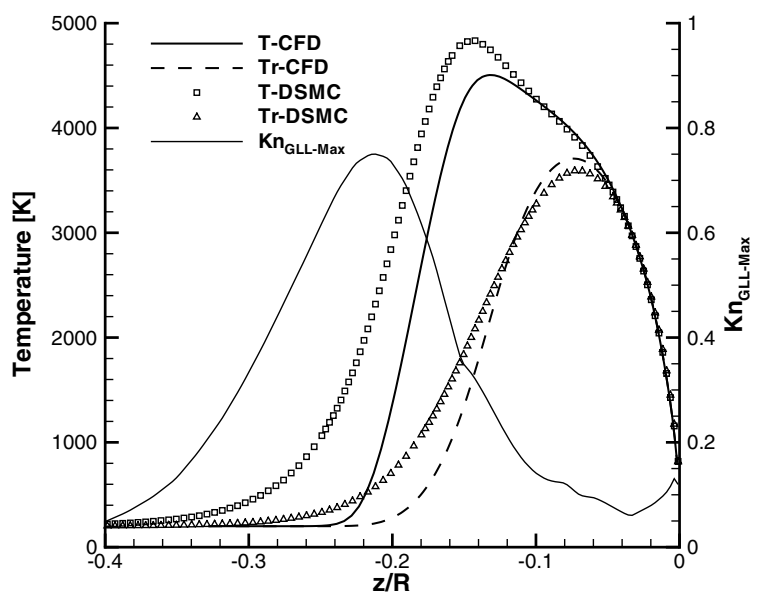

a) Sphere

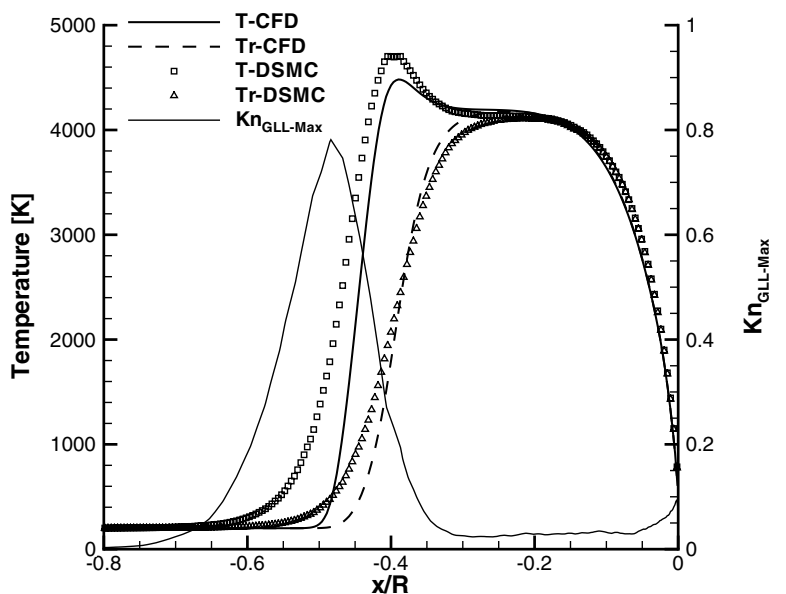

b) Cylinder

Fig. $17 K n_{\infty}=0.01$ : temperature and $K n_{\text {GLL }}$ profiles along the stagnation streamline at Mach 10 . 
of this study is on the sphere, only one case is employed to make a comparison to the cylinder. The case chosen for this comparison is the global Knudsen number of 0.01 case at Mach 10. The integrated drag and peak heat flux are given in Tables 11 and 12, respectively. The tables give the values predicted by DSM $\bar{C}$ and $\overline{C F D}$ along with a percent difference between DSMC and CFD. Once again, it is assumed that the DSMC results are more accurate, so that the percent difference is calculated using the DSMC result. For both the peak heat flux and the integrated drag, the axisymmetric case has much larger differences between the two methods.

From the integrated drag and the total heat flux it can be seen that running a simulation axisymmetrically leads to larger differences between DSMC and CFD. And so the question becomes, how does axisymmetry affect continuum breakdown? This question can be answered by comparing the plots of $K n_{\mathrm{GLL}}$ for the sphere and cylinder cases, as presented in Fig. 16. This figure shows contours of $K n_{\text {GLL }}$ for DSMC and CFD for the cylinder and sphere cases. It is seen that the DSMC prediction of $K n_{\mathrm{GLL}}$ has a much larger amount of continuum breakdown in the wake of the sphere than for the cylinder. It is interesting to note that the amount of breakdown in the shock is approximately the same. The CFD prediction of $K n_{\mathrm{GLL}}$ remains nearly the same for both the sphere and the cylinder. The shock in the cylinder case is farther out than for the sphere, but this is expected due to the ability of gas to flow in all directions around the sphere, thus allowing the shock to move closer to the surface.

Figure 17 gives the temperature and $K n_{\mathrm{GLL}}$ profiles along the stagnation streamline for the sphere and the cylinder. From this figure it is seen that the flow is in continuum breakdown from the shock to the surface for the sphere, whereas for the cylinder there is only breakdown in the shock and in the boundary layer. Because there is less breakdown for the cylinder case, the temperature profiles for CFD and DSMC match very well. The only difference in the temperature profiles for the cylinder is that the shock is thicker in DSMC so that the temperatures begin increasing slightly farther upstream than CFD. Also, notice that the rotational temperature predicted by CFD does not overshoot the DSMC solution as it does with the sphere. Once again this is due to less continuum breakdown between the shock and the surface of the cylinder.

\section{Conclusions}

This study investigated the effects of continuum breakdown on the surface aerothermodynamic properties (pressure, stress, heat transfer rate) of a sphere in Mach 10, 25, and 45 flows of nitrogen gas in regimes varying from continuum to a rarefied gas flow. The first part of this paper focused on the effects of increasing global Knudsen number on continuum breakdown and the surface properties. The differences between CFD and DSMC for peak heat flux and the integrated drag increased for global Knudsen numbers increasing from 0.002 to 0.25 . When the slip boundary conditions were used in the CFD code the agreement between DSMC and CFD improved. It was observed that with increasing global Knudsen number, the amount of continuum breakdown increased and this had a large effect on the surface properties of the sphere. The second part of this study was concerned with the effects of increasing Mach number on continuum breakdown and the surface properties. As Mach number was increased, the amount of continuum breakdown also increased, but there were no clear effects on the surface properties. Even though the amount of continuum breakdown increased with Mach number, the value of $K n_{\mathrm{GLL}}$ remained relatively constant causing the differences in the surface properties between CFD and DSMC to be nearly unchanged. The slip boundary conditions improved the agreement between CFD and DSMC for the three Mach numbers. The last part of this study compared the results of the sphere with the analogous case of a 2-D cylinder for a global Knudsen number of 0.01 at Mach 10. The integrated drag and peak heat flux both display that the differences between CFD and DSMC increase when run axisymmetrically. For DSMC, the amount of continuum breakdown increased when run axisymmetrically as compared with the 2-D case; however, this trend is not observed in CFD. It is clear that continuum breakdown affects surface properties, but the effects are much less noticeable on the surface pressure than on shear stress or heat flux. From this study it has been observed that varying the global Knudsen number, Mach number, and running the simulation axisymmetrically all have an effect on the amount of continuum breakdown. Running the CFD simulations with a slip boundary condition improved the agreement between CFD and DSMC, allowing CFD to more accurately predict the surface properties at higher global Knudsen numbers.

\section{Acknowledgments}

This work is sponsored under a NASA graduate student researchers program fellowship (NNX07AV91H) through NASA Ames Research Center monitored by Michael Wright. The use of supercomputing resources from the University of Michigan and NASA was essential to this investigation and is greatly appreciated.

\section{References}

[1] Vincenti, W. G., and Kruger, C. H., Introduction to Physical Gas Dynamics, Krieger, Malabar, FL, 1965.

[2] Bird, G. A., Molecular Gas Dynamics and the Direct Simulation of Gas Flows, Oxford Univ. Press, Oxford, UK/New York, 1994.

[3] Bird, G. A., "Breakdown of Translational and Rotational Equilibrium in Gaseous Expansions," AIAA Journal, Vol. 8, No. 11, 1970, pp. 19982003.

doi: $10.2514 / 3.49883$

[4] Boyd, I. D., Chen, G., and Chandler, G., "Predicting Failure of the Continuum Fluid Equations in Transitional Hypersonic Flows," Physics of Fluids, Vol. 7, 1995, pp. 210-219.

doi: $10.1063 / 1.868720$

[5] Camberos, J. A., Schrock, C. R., McMullan, R. J., and Branam, R. D., "Development of Continuum Onset Criteria with Direct Simulation Monte-Carlo Using Boltzmann's H-Theorem: Review and Vision," AIAA Paper 2006-2942,5-8 June 2006.

[6] Tiwari, S., "Coupling of the Boltzmann and Euler Equations with Automatic Domain Decomposition," Journal of Computational Physics, Vol. 144, 1998, pp. 710-726. doi:10.1006/jcph.1998.6011

[7] Garcia, A. L., Bell, J. B., Crutchfield, W. Y., and Alder, B. J., "Adaptive Mesh and Algorithm Refinement Using Direct Simulation Monte Carlo," Journal of Computational Physics, Vol. 154, 1999, pp. 134-155. doi:10.1006/jcph.1999.6305

[8] Lofthouse, A. J., Boyd, I. D., and Wright, M. J., "Effects of Continuum Breakdown on Hypersonic Aerothermodynamics," Physics of Fluids, Vol. 19, 2007.doi: $10.1063 / 1.2710289$

[9] Lofthouse, A. J., Scalabrin, L. C., and Boyd, I. D., "Velocity Slip and Temperature Jump in Hypersonic Aerothermodynamics," Journal of Thermophysics and Heat Transfer, Vol. 22, No. 1, 2008, pp. 38-48. doi:10.2514/1.31280

[10] Lofthouse, A. J., Scalabrin, L. C., and Boyd, I. D., "Hypersonic Aerothermodynamics Analysis Across Nonequilibrium Regimes Using Continuum and Particle Methods," AIAA Paper 2007-3903, 2528 June 2007.

[11] Dietrich, S., and Boyd, I. D., "Scalar and Parallel Optimized Implementation of the Direct Simulation Monte Carlo Method," Journal of Computational Physics, Vol. 126, 1996, pp. 328-342. doi:10.1006/jcph.1996.0141

[12] Kannenberg, K. C., and Boyd, I. D., "Strategies for Efficient Particle Resolution in the Direct Simulation Monte Carlo Method," Journal of Computational Physics, Vol. 157, 2000, pp. 727-745. doi:10.1006/jcph.1999.6397

[13] Scalabrin, L. C., and Boyd, I. D., "Development of an Unstructured Navier-Stokes Solver for Hypersonic Nonequilibrium Aerothermodynamics," AIAA Paper 2005-5203,June 2005.

[14] Scalabrin, L. C., and Boyd, I. D., "Numerical Simulation of Weakly Ionized Hypersonic Flow for Reentry Configurations," AIAA Paper 2006-3773, June 2006.

[15] Schwartzentruber, T. E., Scalabrin, L. C., and Boyd, I. D., "Hybrid Particle-Continuum Simulations of Non-Equilibrium Hypersonic Blunt Body FlowFields," AIAA Paper 2006-3602, June 2006.

[16] Boyd, I. D., "Analysis of Rotational Nonequilibrium in Standing Shock Waves of Nitrogen," AIAA Journal, Vol. 28, 1990, pp. 1997-1999. doi: $10.2514 / 3.10511$.

[17] Parker, J. G., "Rotational and Vibrational Relaxation in Diatomic Gases," Physics of Fluids, Vol. 2, 1959, pp. 449-462. doi: $\underline{10.1063 / 1.1724417}$ 
[18] Millikan, R. C., and White, D. R., "Systematics of Vibrational Relaxation," Journal of Chemical Physics, Vol. 39, 1963, pp. 32093213. doi:10.1063/1.1734182

[19] Park, C., "Review of Chemical-Kinetic Problems of Future NASA Missions, 1: Earth Entries," Journal of Thermophysics and Heat Transfer, Vol. 7, No. 3, 1993. doi: $10.2514 / 3.431$

[20] Park, C., Nonequilibrium Hypersonic Aerothermodynamics, Wiley, New York, 1990.

[21] Lumpkin, F. E., Haas, B. L., and Boyd, I. D., "Resolutions of Differences Between Collision Number Definitions in Particle and
Continuum Simulations," Physics of Fluids A, Vol. 3, No. 9, 1991. doi: $10.1063 / 1.857964$

[22] Boyd, I. D., "Analysis of Vibrational Translational Energy Transfer Using the Direct Simulation Monte Carlo Method," Physics of Fluids A, Vol. 3, No. 7, 1991, pp. 1785-1791. doi: $10.1063 / 1.857959$

[23] Gökçen, T., and MacCormack, R. W., "Nonequilibrium Effects for Hypersonic Transitional Flows Using Continuum Approach," AIAA 1989-0461, Jan. 1989.

[24] Gombosi, T. I., Gaskinetic Theory, Cambridge Univ. Press, Cambridge, England, UK, 1994 